\title{
STABILITY ANALYSIS OF EXPLICIT ENTROPY VISCOSITY METHODS FOR NON-LINEAR SCALAR CONSERVATION EQUATIONS
}

\author{
ANDREA BONITO, JEAN-LUC GUERMOND, AND BOJAN POPOV
}

\begin{abstract}
We establish the $L^{2}$-stability of an entropy viscosity technique applied to nonlinear scalar conservation equations. First- and second-order explicit time-stepping techniques using continuous finite elements in space are considered. The method is shown to be stable independently of the polynomial degree of the space approximation under the standard CFL condition.
\end{abstract}

\section{INTRODUCTION}

Owing to a classical theorem by Godunov, it is now well understood that nonlinear approximation is required to approximate solutions of first-order hyperbolic equations with higher-order accuracy (i.e., larger than first-order). One can roughly distinguish two categories of nonlinear methods; the first one uses limiters and nonoscillatory reconstructions; see for example [12,14, 20] and the second one uses nonlinear viscosities [4, 15, 18, 22, 24]. (This categorization is fuzzy as observed in Remark 4.1 of [4.) The purpose of this paper is to analyze the stability properties of a method of the second category which we call entropy viscosity. This method has been introduced in [9, 11] and is based on a research program exposed in [8].

The entropy viscosity technique is a new class of high-order numerical methods for approximating conservation equations. This approach does not use any flux or slope limiters, applies to equations or systems supplemented with one or more entropy inequalities and is easy to implement on a large variety of meshes and polynomial approximations. The use of limiters and nonoscillatory reconstructions is avoided by adding a degenerate nonlinear dissipation to the numerical discretization of the equation or system at hand. The numerical viscosity is set to be proportional to the local size of an entropy production. Scalar conservation equations have many entropy pairs and most physical systems have at least one entropy function satisfying an auxiliary entropy inequality. The entropy satisfies a conservation equation in the regions where the solution is smooth and satisfies an inequality in shocks; this inequality then becomes a selection principle for the physically relevant solution. The amount of violation of the entropy equation is called entropy production. By

Received by the editor January 27, 2012 and, in revised form, October 12, 2012.

2010 Mathematics Subject Classification. Primary 35F25, 65M12, 65N30, 65N22.

Key words and phrases. Nonlinear conservation equations, finite elements, entropy, viscous approximation, stability, time stepping, strong stability preserving time stepping, Runge-Kutta.

This material is based upon work supported by the Department of Homeland Security under agreement 2008-DN-077-ARI018-02, National Science Foundation grants DMS-0811041, DMS0914977, DMS-1015984, AF Office of Scientific Research grant FA99550-12-0358, and is partially supported by award KUS-C1-016-04, made by King Abdullah University of Science and Technology (KAUST) . 
making the numerical diffusion proportional to the entropy production, the numerical dissipation becomes large in the regions of shock and small in the regions where the solution remains smooth.

The method has been implemented with Fourier approximation in [9], with spectral finite elements in [10], with continuous finite elements in [11, with discontinuous finite elements in [26] and various entropy functionals. The method seems to perform well on various benchmarks for a large class of approximation techniques but no theoretical result has yet been produced so far to justify the performance of the method. The present paper is our very first attempt in this direction.

The convergence analysis of nonlinear schemes for conservation equations is complicated even for the one-dimensional linear transport equation. For instance, it was only recently that the convergence rate of the second-order Nessyahu-Tadmor scheme [20] was shown to be better than that of a first-order monotone scheme for the linear transport equation in one space dimension [21]. In the present paper we restrict ourselves to the $L^{2}$-stability of the entropy viscosity method applied to scalar nonlinear conservation equations with various explicit time-stepping techniques using continuous finite elements in space of any degree.

The paper is organized as follows. The problem and the discrete setting at hand are described in \$2 The stability of the first-order forward Euler method using a formally second-order viscosity based on the quadratic entropy $E(u)=\frac{1}{2} u^{2}$ is investigated in $\$ 3$. Two second-order Runge-Kutta (RK2) time stepping techniques are analyzed in $\$ 4$ and $\$ 5$. In $\$ 4$ we focus on the Heun method which is an example of a strong-stability preserving scheme (SSP). Stability is obtained upon adding an entropy viscosity at each step of this two-step method. The viscosity used in the first step depends on the solution from the previous time interval. We prove $L^{2}$-stability using the linear entropy $E(u)=u$, i.e., the entropy equation is the residual of the conservation equation. In $\$ 5$ we analyze the midpoint scheme using again the linear entropy $E(u)=u$ to construct the viscosity. The particularity of this two-step method is that the entropy viscosity is built on the fly; i.e., it is added only at the second step and uses the solution from the first step. This feature could be useful when adaptive refinement is performed. Concluding remarks and numerical illustrations are reported in 86 . The three key results from this paper are Theorem 3.1, Theorem 4.1 and Theorem 5.1.

\section{Preliminaries}

We describe in this section the functional setting used in this paper and we establish preliminary results.

2.1. The scalar conservation equation. Let $\Omega \subset \mathbb{R}^{d}, d \geq 1$, be an open connected domain with Lipschitz boundary. The outward unit normal of $\Omega$ is denoted by $\mathbf{n}$. We consider the scalar-valued conservation equation

$$
\partial_{t} u+\nabla \cdot \mathbf{f}(u)=0, \quad u(x, 0)=u_{0}(x), \quad(x, t) \in \Omega \times \mathbb{R}_{+},
$$

where $\mathbf{f} \in \mathcal{C}^{1}\left(\mathbb{R} ; \mathbb{R}^{d}\right)$. The uniform Lipschitz condition on the flux might seem to be restrictive. For instance, to be useful this condition requires uniform a priori bounds on the discrete solution when $f(v)=\frac{1}{2} v^{2}$. However, since the solution $u$ of (2.1) satisfies such uniform bounds, say

$$
m:=\underset{y \in \Omega}{\operatorname{essinf}} u_{0}(y) \leq u(x, t) \leq \underset{y \in \Omega}{\operatorname{ess} \sup } u_{0}(y)=: M, \quad \forall(x, t) \in \Omega \times(0, T),
$$


a standard way to bypass the uniformly Lipschitz condition at the discrete level consists of replacing $\mathbf{f}$ by $\widetilde{\mathbf{f}}$ so that $\widetilde{\mathbf{f}}(v)=\mathbf{f}(v)$ for all $v \in[m, M]$ and $\widetilde{\mathbf{f}}^{\prime}(v)=\mathbf{f}^{\prime}(m)$ when $v \in(-\infty, m]$ and $\widetilde{\mathbf{f}}^{\prime}(v)=\mathbf{f}^{\prime}(M)$ when $v \in[M, \infty)$.

To avoid boundary condition issues that can be very difficult to handle, we assume that there exists some time $T>0$ so that

$$
\int_{\Omega} u(\mathbf{x}, t) \nabla \cdot \mathbf{f}(u(\mathbf{x}, t)) \mathrm{d} \mathbf{x} \geq 0, \quad \forall t \in[0, T) .
$$

Note that provided $\mathbf{f} \in \mathcal{C}^{1}\left(\mathbb{R} ; \mathbb{R}^{d}\right),(2.3)$ is just the requirement that $\int_{\Omega} \nabla \cdot \mathbf{G}(u) \mathrm{d} \mathbf{x} \geq$ 0 where $\mathbf{G}(u):=\int_{0}^{u} v \mathbf{f}^{\prime}(v) \mathrm{d} v$ is the entropy flux associated with the entropy $E(u)=$ $\frac{1}{2} u^{2}$ (see below). This condition holds with $T=+\infty$ if the boundary conditions are periodic. It also holds if the initial data is compactly supported, and in this case $T$ is the time at which the domain of dependence of $u_{0}$ reaches the boundary of $\Omega$. Dealing with the general case can be done by enforcing entropy compatible boundary conditions à la Bardos, Leroux, and Nédélec [1, instead of condition (2.3). We choose not to take this path to avoid additional technicalities.

It is known that the scalar-valued Cauchy problem (2.1) may have infinitely many weak solutions, but only one of them is physical and satisfies the additional inequalities

$$
\partial_{t} E(u)+\nabla \cdot \mathbf{F}(u) \leq 0,
$$

for all strictly convex functions $E \in \mathcal{C}^{1}(\mathbb{R} ; \mathbb{R})$, where $\mathbf{F}(u):=\int E^{\prime}(v) \mathbf{f}^{\prime}(v) \mathrm{d} v$; see [19]. This physical solution is henceforth called the entropy solution. The function $E(u)$ is called entropy and $\mathbf{F}(u)$ is the associated entropy flux. The most wellknown entropy pairs are the Kružkov pairs generated by $\{E(u)=|u-c|, c \in \mathbb{R}\}$. It is also known for strictly convex fluxes in one space dimension that if the entropy inequality (2.4) holds for one entropy pair and one weak solution $u$ (provided the entropy $E$ is strictly convex), then it also holds for all possible pairs and $u$ is the unique entropy solution.

The objective of this paper is to perform the $L^{2}$-stability analysis of the entropy viscosity method applied to the nonlinear conservation equation (2.1) with forward Euler time stepping and with RK2 time stepping using continuous finite elements in space.

2.2. Functional spaces. We call a mesh $\mathcal{T}$ a subdivision of $\Omega$ into disjoint and closed elements $K$ such that $\bar{\Omega}=\bigcup_{K \in \mathcal{T}} K ; \bar{\Omega}$ is the closure of $\Omega$. The mesh is assumed to be affine to avoid unnecessary technicalities, i.e., $\Omega$ is assumed to be a polygon in two space dimensions or a polyhedron in three space dimensions. For any $K \in \mathcal{T}$, we denote by $h_{K}=\operatorname{diam}(K)$ the diameter of $K$ and by $\rho_{K}$ the diameter of the largest ball inscribed in $K$. Also, we denote $h_{\mathcal{T}}: \Omega \rightarrow \mathbb{R}$ the meshsize function defined by

$$
\left.h_{\mathcal{T}}\right|_{K}:=h_{K}, \quad K \in \mathcal{T} .
$$

The subscript $\mathcal{T}$ is omitted when no confusion is possible. We suppose that we have at hand a family of meshes $\left\{\mathcal{T}_{i}\right\}_{i=1}^{\infty}$ and that this family is shape-regular, meaning that the quantity

$$
c_{s}:=\sup _{i \geq 1} \max _{K \in \mathcal{T}_{i}} h_{K} / \rho_{K}
$$

is finite, i.e., the elements are not too flat. For all $K \in \mathcal{T}_{i}$, the collection of elements in $\mathcal{T}_{i}$ that touch $K$ is denoted $\Delta_{K}$. We assume also that the mesh family is locally 
quasi-uniform in the sense that the quantity

$$
c_{u}:=\sup _{i \geq 1} \max _{K \in \mathcal{T}_{i}}\left(h_{K} /\left(\min _{K^{\prime} \in \Delta_{K}} h_{K^{\prime}}\right)\right)
$$

is finite, i.e., all the elements that touch $K$ have diameters of order $h_{K}$.

Given a mesh $\mathcal{T}$, we define $\mathbb{V}(\mathcal{T})$ the space of piecewise polynomials by

$$
\mathbb{V}(\mathcal{T}):=\left\{V \in \mathcal{C}^{0}(\bar{\Omega}):\left.V\right|_{K} \in \mathcal{P}(K), \forall K \in \mathcal{T}\right\}
$$

where the local finite-dimensional space $\mathcal{P}(K)$ is assumed to contain the multivariate polynomials of total degree at most $k \geq 1$ over $K$, where $k$ is a fixed integer. As a general rule, we will use capital letters to denote discrete functions. Finally, the $L^{2}$-scalar product over a domain $S \subset \mathcal{T}$ is denoted by $(\cdot, \cdot)_{S}$, and we abuse the notation by using $(\cdot, \cdot)_{\Omega}$ instead of $(\cdot, \cdot)_{\mathcal{T}}$. We often use the shorter notation $\|\cdot\|_{L^{2}}$ for $\|\cdot\|_{L^{2}(\Omega)}$ whenever it is unambiguous to do so.

We denote $\Pi_{\mathcal{T}}^{0}$ the $L^{2}$-projection onto constants, i.e., $\left.\Pi_{\mathcal{T}}^{0} \varphi\right|_{K}:=\frac{1}{|K|} \int_{K} \varphi$ for $K \in \mathcal{T}$, and $\Pi_{\mathcal{T}}$ the $L^{2}$-projection onto $\mathbb{V}(\mathcal{T})$. We will frequently use the following inverse inequality

$$
\begin{array}{rlrl}
h_{K}^{-\frac{1}{2}}\|V\|_{L^{2}(\partial K)}+\|\nabla V\|_{L^{2}(K)} & \leq c_{i} h_{K}^{-1}\|V\|_{L^{2}(K)}, & & \forall V \in \mathbb{V}(\mathcal{T}), \quad \forall K \in \mathcal{T}, \\
\|V\|_{L^{\infty}(K)} \leq c_{\infty}|K|^{-\frac{1}{2}}\|V\|_{L^{2}(K)}, & \forall V \in \mathbb{V}(\mathcal{T}), \quad \forall K \in \mathcal{T},
\end{array}
$$

and approximation estimate

$$
\left\|v-\Pi_{\mathcal{T}}^{0} v\right\|_{L^{2}(\Omega)} \leq c_{0}\left\|h_{\mathcal{T}} \nabla v\right\|_{L^{2}(\Omega)}, \quad \forall v \in H^{1}(\Omega) .
$$

The above constants $c_{i}, c_{\infty}, c_{0}$ solely depend on the polynomial degree $k$, the domain $\Omega$ and the mesh shape regularity constant $c_{u}$ and $c_{s}$ defined in (2.5)-(2.6). In the rest of this manuscript, $c, c^{\prime}, c^{\prime \prime}$ denote generic constants that may depend solely on the above constants if not stated otherwise. In order to simplify the presentation, we shall explicitly mention the specific constants only after the step invoking the corresponding estimate. When confusion is not possible, we omit the dependency in $\mathcal{T}$ using the abbreviation $\Pi:=\Pi_{\mathcal{T}}, \Pi^{0}:=\Pi_{\mathcal{T}}^{0}$ and $h:=h_{\mathcal{T}}$.

For any subset $S \subset \mathcal{T}$ we define the two sets $\bar{S}$ and $\dot{S}$ as

$$
\begin{aligned}
& \bar{S}:=\bigcup_{K \in S} \Delta_{K}=\left\{K^{\prime} \in \mathcal{T}: \exists K \in S, K^{\prime} \cap K \neq \emptyset\right\}, \\
& \dot{S}:=\mathcal{T} \backslash(\overline{\mathcal{T} \backslash S}) .
\end{aligned}
$$

The set $\bar{S}$ is composed of $S$ plus the layer of elements surrounding $S$ (not to be confused with the closure of $S$ ). The set $\dot{S}$ is the complement in $\mathcal{T}$ of $\overline{S^{c}}$, where $S^{c}:=\mathcal{T} \backslash S$ (not to be confused with the interior of $S$ ).

For all subsets $S \subset \mathcal{T}$, we define the restriction operator $\mathcal{R}_{S}: \mathbb{V}(\mathcal{T}) \longrightarrow \mathbb{V}(\mathcal{T})$ as follows. Let $\left\{\psi_{1}, \ldots, \psi_{M}\right\}$ be the global shape functions spanning $\mathbb{V}(\mathcal{T})$. Let $I$ be the set of indices, $i$, so that the support of $\psi_{i}$ has a nonempty intersection with $\dot{S}$ for all $i \in I$. Then for all $V:=\sum_{i=1}^{M} V_{i} \psi_{i} \in \mathbb{V}(\mathcal{T})$, we set $\mathcal{R}_{S} V=\sum_{i \in I} V_{i} \psi_{i}$. This definition implies that

$$
\mathcal{R}_{S} V \in \mathbb{V}(\mathcal{T}), \quad \text { and } \quad \mathcal{R}_{S} V(\mathbf{x}):= \begin{cases}0 & \text { if } \mathbf{x} \in S^{c}:=\mathcal{T} \backslash S \\ V(\mathbf{x}) & \text { if } \mathbf{x} \in \dot{S}\end{cases}
$$


Lemma 2.1. There is a uniform constant $c_{\mathcal{R}}$ depending on $c_{\infty}$ and the polynomial degree $k$ so that the following holds:

$$
\left\|\mathcal{R}_{S} V\right\|_{L^{2}(S \backslash \dot{S})} \leq c_{\mathcal{R}}\|V\|_{L^{2}(S \backslash \dot{S})}, \quad \forall V \in \mathbb{V}(\mathcal{T}), \quad \forall S \subset \mathcal{T}
$$

Proof. Let $K \in S \backslash \dot{S}$. Using (2.9) and the definition of $\mathcal{R}_{\mathcal{T}} V$ we infer that

$$
\left\|\mathcal{R}_{S} V\right\|_{L^{2}(K)} \leq|K|^{1 / 2}\left\|\mathcal{R}_{S} V\right\|_{L^{\infty}(K)} \leq c|K|^{1 / 2}\|V\|_{L^{\infty}(K)} \leq c c_{\infty}\|V\|_{L^{2}(K)} .
$$

The desired result follows readily.

\section{Forward Euler stability}

We approximate in time the nonlinear conservation equation (2.1) using the first-order forward Euler method and we establish the $L^{2}$-stability of the method.

3.1. The algorithm. Let $\mathcal{T}$ be a mesh and let $U^{0} \in \mathbb{V}(\mathcal{T})$ be an approximation of $u_{0}$. Let us set $\delta t_{-1}=+\infty$ and $t_{0}=0$. The forward Euler discretization of the equation (2.1) is constructed as follows. Let $U^{n} \in \mathbb{V}(\mathcal{T})$ be the approximation of $u$ at time $t_{n}, n \geq 0$. To avoid boundary condition issues we assume that the following conservation property holds

$$
\left(\nabla \cdot \mathbf{f}\left(U^{n}\right), U^{n}\right)_{\Omega} \geq 0 .
$$

As mentioned in 2.1, this property is known to hold if $u_{0}$ is compactly supported and $t_{n}$ is small; it also holds if the boundary conditions are periodic.

Let $c_{\tau} \geq 1$ be a number and let $\lambda>0$ be another positive number that we henceforth call the CFL number; we select the time step $\delta t_{n}$ so that

$$
\delta t_{n} \leq \min \left(\lambda \min _{K \in \mathcal{T}} \frac{h_{K}}{\left\|\mathbf{f}^{\prime}\left(U^{n}\right)\right\|_{L^{\infty}(K)}}, c_{\tau} \delta t_{n-1}\right) .
$$

Note that the quantity $\min _{K \in \mathcal{T}} \frac{h_{K}}{\left\|\mathbf{f}^{\prime}\left(U^{n}\right)\right\|_{L^{\infty}(K)}} \geq \frac{1}{\left\|\mathbf{f}^{\prime}\right\|_{L^{\infty}\left(\mathbb{R} ; \mathbb{R}^{d}\right)}} \min _{K \in \mathcal{T}} h_{K}$ is bounded away from zero since $\mathbf{f}$ is assumed to be uniformly Lipschitz; as a result, it is always possible to select $\delta t_{n}>0$ satisfying (3.2) and to advance in time. The condition $\delta t_{n} \leq c_{\tau} \delta t_{n-1}$ ensures the time stepping is quasi-uniform. Let $t_{n+1}=t_{n}+\delta t_{n}$ and let $U^{n+1} \in \mathbb{V}(\mathcal{T})$ be such that

$$
\left(U^{n+1}-U^{n}+\delta t_{n} \nabla \cdot \mathbf{f}\left(U^{n}\right), V\right)_{\Omega}+\delta t_{n}\left(\nu^{n} \nabla U^{n}, \nabla V\right)_{\Omega}=0, \quad \forall V \in \mathbb{V}(\mathcal{T}),
$$

where $\nu^{n}$ is the entropy viscosity that we now define. Three different residuals are used to construct the entropy viscosity $\nu^{n}$. We define the residual of the equation $R^{n}$,

$$
R^{n}:=\frac{U^{n}-U^{n-1}}{\delta t_{n-1}}+\nabla \cdot \mathbf{f}\left(U^{n}\right),
$$

and we define two entropy residuals $R_{E 1}^{n}, R_{E 2}^{n}$,

$$
R_{E 1}^{n}:=R^{n} U^{n}, \quad \text { and } \quad R_{E 2}^{n}:=\frac{E\left(U^{n}\right)-E\left(U^{n-1}\right)}{\delta t_{n-1}}+\mathbf{f}^{\prime}\left(U^{n}\right) \cdot \nabla E\left(U^{n}\right) .
$$

where $E(v)=\frac{1}{2} v^{2}$ is the quadratic entropy. Let $R_{E}^{n}$ be the total entropy residual defined as follows:

$$
\left.R_{E}^{n}\right|_{K}:=\left\|R_{E 1}^{n}\right\|_{L^{\infty}(K)}+\left\|R_{E 2}^{n}\right\|_{L^{\infty}(K)}+\delta t_{n}\left\|R^{n}\right\|_{L^{\infty}(K)}^{2} .
$$

We then define the entropy viscosity over each cell $K$ as follows:

$$
\left.\nu^{n}\right|_{K}:=h_{K} \min \left(c_{M}\left\|\mathbf{f}^{\prime}\left(U^{n}\right)\right\|_{L^{\infty}(K)},\left.c_{E} R_{E}^{n}\right|_{K}\right),
$$


where $c_{M}>0$ and $c_{E}>0$ are user-defined constants.

Remark 3.1 (Choice of Parameters). Usually we take $c_{M}=\frac{1}{2 k}$ in one space dimension and $c_{M}=\frac{1}{4 k}$ in two space dimensions (recall that $k$ is the polynomial degree used in the local approximation space $\mathcal{P}$ ). The constant $c_{E}$ is dimensional and is also user-defined; for instance, it can be defined as follows:

$$
c_{E}:=\mathfrak{c}_{E} \frac{D}{\frac{1}{|\Omega|} \int_{\Omega}\left|E\left(U^{0}\right)\right|}
$$

or, equivalently, $c_{E}:=\mathfrak{c}_{E}|\Omega|\left\|\nabla E\left(U^{0}\right)\right\|_{L^{1}(\Omega)}^{-1}$, or $c_{E}:=\mathfrak{c}_{E} D\left\|E\left(U^{0}\right)\right\|_{L^{\infty}(\Omega)}^{-1}$, where $|\Omega|:=\operatorname{meas}(\Omega), D:=\operatorname{diam}(\Omega)$ and $\mathfrak{c}_{E}$ is a nondimensional constant of order one.

Remark 3.2 (Consistency of the entropy residual). Note that $R_{E}$ is formally firstorder, $\mathcal{O}\left(\delta t_{n}+h_{K}^{k}\right)$, in the region where $u$ is smooth. That is, the entropic viscosity is formally second-order, i.e., $\mathcal{O}\left(h_{K}\left(\delta t_{n}+h_{K}^{k}\right)\right)$, which is greater than the overall consistency order of the first-order Euler method. As a result, we expect the method to be as accurate as the first-order Euler method for smooth solutions, i.e., the error should be formally $\mathcal{O}\left(\delta t+h^{k}\right)$ in $L^{p}$-norms, $1 \leq p<\infty$, provided some stability is established.

The entropic viscosity naturally splits the mesh $\mathcal{T}$ into a viscous and a smooth set as follows:

$$
\mathcal{T}=\mathcal{T}_{V}^{n} \cup \mathcal{T}_{S}^{n}, \quad\left\{\begin{array}{l}
\mathcal{T}_{V}^{n}:=\left\{K \in \mathcal{T}:\left.\nu^{n}\right|_{K}=c_{M} h_{K}\left\|\mathbf{f}^{\prime}\left(U^{n}\right)\right\|_{L^{\infty}(K)}\right\}, \\
\mathcal{T}_{S}^{n}:=\mathcal{T} \backslash \mathcal{T}_{V}^{n}:=\left\{K \in \mathcal{T}:\left.\nu^{n}\right|_{K}=\left.c_{E} h_{K} R_{E}\right|_{K}\right\} .
\end{array}\right.
$$

This decomposition will arise in the stability analysis below. For the moment, note that no stability issue should arise on $\mathcal{T}_{V}^{n}$ due to the presence of the first-order viscosity $\left.\nu^{n}\right|_{K}=c_{M} h_{K}\left\|\mathbf{f}^{\prime}(U)\right\|_{L^{\infty}(K)}, \forall K \in \mathcal{T}_{V}^{n}$. Establishing stability on $\mathcal{T}_{S}^{n}$ will turn out to be the more technical part of the proof; it will be essential to observe that the discrete time derivative satisfies

$$
\left(\frac{U^{n}-U^{n-1}}{\delta t_{n}}\right)^{2}=2 \frac{R_{E 1}^{n}-R_{E 2}^{n}}{\delta t_{n}} \leq 2 \frac{\left|R_{E 1}^{n}\right|+\left|R_{E 2}^{n}\right|}{\delta t_{n}},
$$

which justifies the introduction on the two entropy residuals $R_{E 1}^{n}$ and $R_{E 2}^{n}$.

3.2. Stability analysis of forward Euler. We are now in position to prove the stability estimate for the forward Euler scheme (3.3).

Theorem 3.1 (Stability of the Forward-Euler Scheme). Assume that the conditions (3.1)-(3.2) are satisfied. There is $\Lambda_{0}>0$ that depends only on the user-defined parameters $c_{M}, \mathfrak{c}_{E}$, the Lipschitz constant of the flux, and on the mesh family constants $c_{0}, c_{i}$, and there is a constant $c$ that additionally depends linearly on the final time $T$ so that the solution to (3.3) satisfies the following $L^{2}$-stability estimate for all $\lambda \leq \Lambda_{0}$ :

$$
\left\|U^{n}\right\|_{L^{2}(\Omega)}^{2}+\sum_{i=0}^{n}\left\|\sqrt{\nu^{i}} \nabla U^{i}\right\|_{L^{2}(\Omega)}^{2} \leq\left\|U^{0}\right\|_{L^{2}(\Omega)}^{2}(1+c \lambda), \quad \forall t_{n} \leq T .
$$

Proof. Step 1. Using $V=2 U^{n}$ in (3.3) together with the conservation property (3.1), we obtain

$$
\left\|U^{n+1}\right\|_{L^{2}(\Omega)}^{2}-\left\|U^{n}\right\|_{L^{2}(\Omega)}^{2}+2 \delta t_{n}\left\|\sqrt{\nu^{n}} \nabla U^{n}\right\|_{L^{2}(\Omega)}^{2} \leq\left\|U^{n+1}-U^{n}\right\|_{L^{2}(\Omega)}^{2} .
$$


We now estimate the right-hand side of (3.12). Defining $B:=\left\{V \in \mathbb{V}(\mathcal{T}) \mid\|V\|_{L^{2}(\Omega)}\right.$ $=1\}$, and using $\left.\nu^{n}\right|_{K} \leq c_{M}\left\|\mathbf{f}^{\prime}\left(U^{n}\right)\right\|_{L^{\infty}(K)} h_{K}$, (3.3) yields

$$
\begin{aligned}
\left\|U^{n+1}-U^{n}\right\|_{L^{2}(\Omega)}^{2} & =\sup _{V \in B}\left(U^{n+1}-U^{n}, V\right)_{\Omega}^{2} \\
& \leq 2 \delta t_{n}^{2} \sup _{v \in B}\left(\left(\nabla \cdot \mathbf{f}\left(U^{n}\right), V\right)_{\Omega}^{2}+\left(\nu^{n} \nabla U, \nabla V\right)_{\Omega}^{2}\right) \\
& \leq 2 \delta t_{n}^{2}\left\|\nabla \cdot \mathbf{f}\left(U^{n}\right)\right\|_{L^{2}(\Omega)}^{2}+2 \delta t_{n} c_{M} c_{i}^{2} \lambda\left\|\sqrt{\nu^{n}} \nabla U^{n}\right\|_{L^{2}(\Omega)}^{2} .
\end{aligned}
$$

Therefore we can rewrite (3.12) as follows:

$$
\begin{aligned}
\left\|U^{n+1}\right\|_{L^{2}(\Omega)}^{2}-\left\|U^{n}\right\|_{L^{2}(\Omega)}^{2}+2 \delta t_{n}\left(1-c_{M} c_{i}^{2} \lambda\right)\left\|\sqrt{\nu^{n}} \nabla U^{n}\right\|_{L^{2}(\Omega)}^{2} & \\
& \leq 2 \delta t_{n}^{2}\left\|\nabla \cdot \mathbf{f}\left(U^{n}\right)\right\|_{L^{2}(\Omega)}^{2} .
\end{aligned}
$$

The remainder of the proof consists of estimating a bound on $\left\|\nabla \cdot \mathbf{f}\left(U^{n}\right)\right\|_{L^{2}(\Omega)}^{2}$, and we are going to invoke the partition $\mathcal{T}=\mathcal{T}_{V}^{n} \cup \mathcal{T}_{S}^{n}$ for that purpose.

Step 2 (Control over $\left.\mathcal{T}_{V}^{n}\right)$. The viscosity is large enough to control $\delta t_{n}\left\|\nabla \cdot \mathbf{f}\left(U^{n}\right)\right\|_{L^{2}(\Omega)}$ on the viscous set $\mathcal{T}_{V}^{n}$, and we have:

$$
\begin{aligned}
\delta t_{n}^{2} \int_{\mathcal{T}_{V}^{n}}\left|\nabla \cdot \mathbf{f}\left(U^{n}\right)\right|^{2} \leq\left\|h^{-1} \mathbf{f}^{\prime}\left(U^{n}\right)\right\|_{L^{\infty}(\Omega)} \delta t_{n}^{2} c_{M}^{-1} \int_{\mathcal{T}_{V}^{n}} \nu^{n}\left|\nabla U^{n}\right|^{2} \\
\leq c_{M}^{-1} \delta t_{n} \lambda\left\|\sqrt{\nu^{n}} \nabla U^{n}\right\|_{L^{2}(\Omega)}^{2} .
\end{aligned}
$$

Step 3 (Control over $\mathcal{T}_{S}^{n}$ ). Recalling the bound (3.10), we infer that

$$
\begin{aligned}
\delta t_{n-1}\left|\nabla \cdot \mathbf{f}\left(U^{n}\right)\right| & =\left|\delta t_{n-1} R^{n}-\left(U^{n}-U^{n-1}\right)\right| \\
& \leq \delta t_{n-1}\left|R^{n}\right|+\sqrt{2} \delta t_{n-1}^{\frac{1}{2}}\left(\left|R_{E 1}^{n}\right|^{\frac{1}{2}}+\left|R_{E 2}^{n}\right|^{\frac{1}{2}}\right) .
\end{aligned}
$$

With this estimate in hand we infer that the following estimate holds on the smooth set $\mathcal{T}_{S}^{n}$,

$$
\begin{aligned}
& \delta t_{n}^{2} \int_{\mathcal{T}_{S}^{n}}\left|\nabla \cdot \mathbf{f}\left(U^{n}\right)\right|^{2} \\
& \quad \leq \delta t_{n}^{\frac{3}{2}} \int_{\mathcal{T}_{S}^{n}}\left|\nabla U^{n}\right|\left|\mathbf{f}^{\prime}\left(U^{n}\right)\right|\left(\delta t_{n}^{\frac{1}{2}}\left|R^{n}\right|+\sqrt{2} \delta t_{n}^{\frac{1}{2}} \delta t_{n-1}^{-\frac{1}{2}}\left(\left|R_{E 1}^{n}\right|^{\frac{1}{2}}+\left|R_{E 2}^{n}\right|^{\frac{1}{2}}\right)\right) \\
& \quad \leq c \delta t_{n}^{\frac{3}{2}} \int_{\mathcal{T}_{S}^{n}}\left|\nabla U^{n}\right|\left|\mathbf{f}^{\prime}\left(U^{n}\right)\right|\left(R_{E}^{n}\right)^{1 / 2},
\end{aligned}
$$

where we have used the quasi-uniformity assumption (3.2) of the time stepping. Hence, we obtain

$$
\begin{aligned}
\delta t_{n}^{2}\left\|\nabla \cdot \mathbf{f}\left(U^{n}\right)\right\|_{L\left(\mathcal{T}_{S}^{n}\right)}^{2} \leq & c c_{E}^{-1} \lambda \delta t_{n}\left|\mathcal{T}_{S}^{n}\right|\left\|\mathbf{f}^{\prime}\left(U^{n}\right)\right\|_{L^{\infty}(\Omega)} \\
& +\frac{1}{2} \delta t_{n}^{2} \lambda^{-1} c_{E} \int_{\mathcal{T}_{S}^{n}}\left|\nabla U^{n}\right|^{2}\left|\mathbf{f}^{\prime}\left(U^{n}\right)\right| R_{E}^{n},
\end{aligned}
$$

which after using that $\mathbf{f}$ is uniformly Lipschitz together with the expression of the viscosity $\nu_{K}^{n}=c_{E} h_{K} R_{E}^{n}$ on $\mathcal{T}_{S}^{n}$, leads to

$$
\delta t_{n}^{2}\left\|\nabla \cdot \mathbf{f}\left(U^{n}\right)\right\|_{L^{2}\left(\mathcal{T}_{S}^{n}\right)}^{2} \leq c \lambda g_{E} \delta t_{n}\left\|U^{0}\right\|_{L^{2}(\Omega)}^{2}+\frac{1}{2} \delta t_{n}\left\|\sqrt{\nu^{n}} \nabla U^{n}\right\|_{L^{2}(\Omega)}^{2},
$$

where we set $g_{E}:=\left\|\mathbf{f}^{\prime}\right\|_{L^{\infty}(\mathbb{R})}|\Omega| c_{E}^{-1}\left\|U^{0}\right\|_{L^{2}(\Omega)}^{-2}$. 
Step 4. Setting $\Lambda_{0}:=\frac{c_{M}}{4\left(c_{M}^{2} c_{i}^{2}+1\right)}$ and inserting (3.14) and (3.15) into (3.13), we finally obtain that the following holds for all $\lambda \leq \Lambda_{0}$,

$$
\left\|U^{n+1}\right\|_{L^{2}(\Omega)}^{2}-\left\|U^{n}\right\|_{L^{2}(\Omega)}^{2}+\delta t_{n}\left\|\sqrt{\nu^{n}} \nabla U^{n}\right\|_{L^{2}(\Omega)}^{2} \leq c \lambda g_{E} \delta t_{n},
$$

which immediately leads to

$$
\left\|U^{n}\right\|_{L^{2}(\Omega)}^{2}+\sum_{i=0}^{n}\left\|\sqrt{\nu^{i}} \nabla U^{i}\right\|_{L^{2}(\Omega)}^{2} \leq\left\|U^{0}\right\|_{L^{2}(\Omega)}^{2}\left(1+c \lambda g_{E} t_{n}\right), \quad \forall n \in \mathbb{N} .
$$

Observe that $\lambda g_{E} t_{n}$ is a dimensionless constant. This completes the proof.

\section{Runge-Kutta 2 (Heun)}

We now turn our attention to the second-order RK2/Heun time discretization to approximate (2.1). This time stepping is known to be a Strong-Stability-Preserving method [7]. The viscosity considered in this section is mainly based on the the linear entropy $E(u)$, i.e., the residual of the equation at the previous time step. We analyze another second-order method with the viscosity computed on the fly in $\$ 5$, The present scheme and that in $\$ 5$ do not require the quasi-uniformity assumption that had to be invoked for the forward Euler scheme; see (3.2).

4.1. The algorithm. Let us set $t_{0}=0$ and let $U^{0} \in \mathbb{V}(\mathcal{T})$ be an approximation of $u_{0}$. Let $\lambda>0$ be a CFL number. Let $U^{n} \in \mathbb{V}(\mathcal{T})$ be the approximation of $u$ at time $t_{n}, n \geq 0$. Let $\delta t_{n}$ be a given time step possibly restricted later by the CFL number (see (4.4) ) and set $t_{n+1}=t_{n}+\delta t_{n}$. The fully discrete RK2/Heun algorithm that we consider is formulated as follows: Find $W^{n} \in \mathbb{V}(\mathcal{T})$ and $U^{n+1} \in \mathbb{V}(\mathcal{T})$ satisfying

$$
\begin{gathered}
\left(W^{n}, V\right)_{\Omega}-\left(U^{n}, V\right)_{\Omega}+\delta t_{n}\left(\nabla \cdot \mathbf{f}\left(U^{n}\right), V\right)_{\Omega}+\delta t_{n}\left(\nu_{1}^{n} \nabla U^{n}, \nabla V\right)_{\Omega}=0, \\
\left(U^{n+1}-\frac{1}{2}\left(W^{n}+U^{n}\right), V\right)_{\Omega}+\frac{\delta t_{n}}{2}\left(\nabla \cdot \mathbf{f}\left(W^{n}\right), V\right)_{\Omega}+\frac{\delta t_{n}}{2}\left(\nu_{2}^{n} \nabla W^{n}, \nabla V\right)_{\Omega}=0,
\end{gathered}
$$

for all $V \in \mathbb{V}(\mathcal{T})$, where the viscosities $\nu_{1}^{n}, \nu_{2}^{n}$ are defined below. To avoid issues induced by the boundary condition we assume that both $U^{n}$ and $W^{n}$ satisfy the following conservation properties:

$$
\left(\nabla \cdot \mathbf{f}\left(U^{n}\right), U^{n}\right)_{\Omega} \geq 0, \quad\left(\nabla \cdot \mathbf{f}\left(W^{n}\right), W^{n}\right)_{\Omega} \geq 0 .
$$

We refer to 2.1 for a discussion on the validity of this assumption. We assume that $\delta t_{n}$ satisfies the additional condition

$$
\delta t_{n} \leq \lambda \min _{K \in \mathcal{T}} \frac{h_{K}}{\max \left(\left\|\mathbf{f}^{\prime}\left(U^{n}\right)\right\|_{L^{\infty}(K)},\left\|\mathbf{f}^{\prime}\left(W^{n}\right)\right\|_{L^{\infty}(K)}\right)} .
$$

If this condition is not satisfied at the end of the time step, the computation of $W^{n}$ and $U^{n+1}$ is redone with a smaller time step, say $\delta t_{n}$ is divided by 1.5 . Note that due to the uniform Lipschitz assumption on $\mathbf{f}$, picking $\delta t_{n}$ smaller than $\frac{1}{\left\|\mathbf{f}^{\prime}\right\|_{L^{\infty}(\mathbb{R})}} \min _{K \in \mathcal{T}} h_{K}$ always guarantees that (4.4) holds.

Let us now construct the viscosities $\nu_{1}^{n}, \nu_{2}^{n}$. Let $U^{-1}=U^{0}$, and consider the residual $R^{n}, n \geq 0$, defined by

$$
R^{n}:=\frac{U^{n}-U^{n-1}}{\delta t_{n-1}}+\nabla \cdot \mathbf{f}\left(U^{n}\right) .
$$


Let $c_{M}>0, c_{E}>0, \alpha \geq 0$ be three real numbers and let us consider the partition of $\mathcal{T}$ defined at time step $t_{n}$ as follows:

$$
\mathcal{T}=\mathcal{T}_{V}^{n} \cup \mathcal{T}_{S}^{n}, \quad\left\{\begin{array}{l}
\mathcal{T}_{S}^{n}:=\left\{K \in \mathcal{T}: c_{E} h_{K}^{\alpha}\left\|R^{n}\right\|_{L^{\infty}(K)} \leq c_{M}\left\|\mathbf{f}^{\prime}\left(U^{n}\right)\right\|_{L^{\infty}(K)}\right\}, \\
\mathcal{T}_{V}^{n}:=\mathcal{T}_{h} \backslash \mathcal{T}_{S}^{n} .
\end{array}\right.
$$

We now define the viscosities $\nu_{1}^{n}, \nu_{2}^{n}, n \geq 0$, to be piecewise constant functions on the mesh cells. For any $K \in \mathcal{T}$ we set $\left.\nu_{1}^{0}\right|_{K}=c_{M} h_{K}\left\|\mathbf{f}^{\prime}\left(U^{0}\right)\right\|_{L^{\infty}(K)}$ and for $n \geq 1$, (4.7)

$$
\left.\nu_{1}^{n}\right|_{K}:= \begin{cases}c_{M} h_{K}\left\|\mathbf{f}^{\prime}\left(U^{n}\right)\right\|_{L^{\infty}(K)} & \text { if } K \in \overline{\mathcal{T}_{V}^{n}}, \\ h_{K} \max \left(c_{E} h_{K}^{\alpha}\left\|R^{n}\right\|_{L^{\infty}(K)}, c_{M} \operatorname{Osc}_{K}\left(\mathbf{f}, U^{n}\right)\right) & \text { if } K \in \dot{\mathcal{T}_{S}^{n}}:=\mathcal{T} \backslash \overline{\mathcal{T}_{V}^{n}},\end{cases}
$$

where

$$
\operatorname{osc}_{K}\left(\mathbf{f}, U^{n}\right):=\frac{\left\|\nabla \cdot \mathbf{f}\left(U^{n}\right)-\Pi^{0}\left(\nabla \cdot \mathbf{f}\left(U^{n}\right)\right)\right\|_{L^{\infty}(K)}^{2}}{4\left\|\mathbf{f}^{\prime}\left(U^{n}\right)\right\|_{L^{\infty}(K)}\left\|\nabla U^{n}\right\|_{L^{\infty}(K)}^{2}} .
$$

Note that $\operatorname{osc}_{K}\left(\mathbf{f}, U^{n}\right) \leq\left\|\mathbf{f}^{\prime}\left(U^{n}\right)\right\|_{L^{\infty}(K)}$. The second sub-step viscosity $\nu_{2}^{n}$ is defined as follows for all $n \geq 0$ :

$$
\begin{aligned}
\left.\nu_{2}^{n}\right|_{K} & :=c_{M} h_{K} \operatorname{nl}_{K}\left(\mathbf{f}, W^{n}, U^{n}\right), \\
\mathrm{nl}_{K}\left(\mathbf{f}, W^{n}, U^{n}\right) & :=\frac{1}{2} \frac{\left\|\mathbf{f}^{\prime}\left(W^{n}\right)-\mathbf{f}^{\prime}\left(U^{n}\right)\right\|_{L^{\infty}(K)}^{2}}{\left\|\mathbf{f}^{\prime}\left(W^{n}\right)|+| \mathbf{f}^{\prime}\left(U^{n}\right) \mid\right\|_{L^{\infty}(K)}} .
\end{aligned}
$$

Several comments are in order regarding the definition of the viscosities.

Remark 4.1 (Oscillation of $\nabla \cdot \mathbf{f}\left(U^{n}\right)$ ). The oscillation of $\nabla \cdot \mathbf{f}\left(U^{n}\right)$, denoted $\operatorname{osc}_{K}\left(\mathbf{f}, U^{n}\right)$, and the nonlinear variation of $\mathbf{f}$, denoted $\mathrm{nl}_{K}\left(\mathbf{f}, W^{n}, U^{n}\right)$, are both zero for the linear transport equation, $\mathbf{f}(u):=\boldsymbol{\beta} u, \boldsymbol{\beta} \in \mathbb{R}^{d}$. The purpose of these two terms is to help control the nonlinearity of the flux. To the best of our knowledge, stability under the usual CFL condition of the Heun discretization of the linear transport equation with continuous finite elements is known so far only for the piecewise linear approximation [5. This issue with the piecewise linear approximation does not seem to arise for higher-order time stepping [5, 25. The oscillation term $\operatorname{osc}_{K}\left(\mathbf{f}, U^{n}\right)$ in the definition of $\nu_{1}^{n}$ seems to be necessary to handle finite elements of polynomial degrees larger than one.

Remark 4.2 (Alternative Expression of $\nu_{1}^{n}$ ). The viscosity $\nu_{1}^{n}$ can be rewritten in the alternative form

$$
\left.\nu_{1}^{n}\right|_{K}:=h_{K} \min \left(c_{M}\left\|\mathbf{f}^{\prime}\left(U^{n}\right)\right\|_{L^{\infty}(K)}, \max \left(c_{E} h_{K}^{\alpha}\left\|R^{n}\right\|_{L^{\infty}(K)}, c_{M} \operatorname{Osc}_{K}\left(\mathbf{f}, U^{n}\right)\right)\right),
$$

for all $K \in \mathcal{T}_{V}^{n} \cup \dot{\mathcal{T}}_{S}^{n}, n \geq 1$, and $\left.\nu_{1}^{n}\right|_{K}:=c_{M} h_{K}\left\|\mathbf{f}^{\prime}\left(U^{n}\right)\right\|_{L^{\infty}(K)}$, for $K \in \mathcal{L}^{n}$, where we have defined $\mathcal{L}^{n}:=\mathcal{T}_{S}^{n} \backslash \dot{\mathcal{T}}_{S}^{n}$. The viscosity saturates to first-order in the so-called viscous set $\mathcal{T}_{V}^{n} \cup \mathcal{L}^{n}$ and is small in the so-called smooth set $\dot{\mathcal{T}}_{S}^{n}$; see Figure 1

Remark 4.3 (Consistency of viscosities). Note that the terms $c_{M} h_{K} \operatorname{osc}_{K}\left(\mathbf{f}, U^{n}\right)$ and $c_{M} h_{K}\left|R^{n}\right|$ are formally $\mathcal{O}\left(h_{K}^{3}\right)$ and $\mathcal{O}\left(h_{K}^{1+\alpha}\left(\delta t_{n}+h_{K}^{k}\right)\right)$, respectively. This mean that the viscosity $\left.\nu_{1}\right|_{K}$ is $\mathcal{O}\left(h_{K}^{2+\alpha}\right)$ under the CFL condition. The viscosity $\left.\nu_{2}\right|_{K}=c_{M} h_{K} \mathrm{nl}_{K}\left(\mathbf{f}, W^{n}, U^{n}\right)$ is formally $\mathcal{O}\left(\delta t_{n}^{2} h_{K}\right)$, i.e., it is third-order in the smooth region $\dot{\mathcal{T}}_{S}^{n}$. Overall the consistency order of the artificial viscosities is higher 


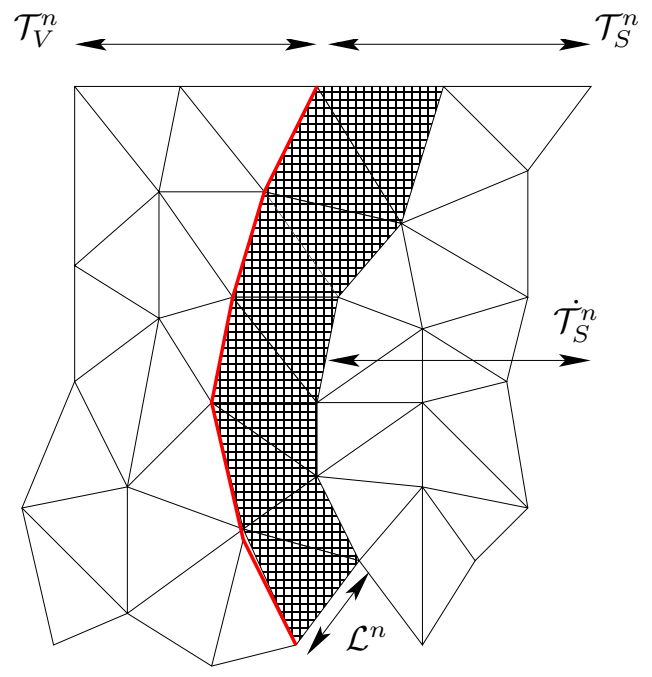

FIGURE 1. Schematic representation of the partition $\mathcal{T}=\dot{\mathcal{T}}_{S}^{n} \cup$ $\mathcal{L}^{n} \cup \mathcal{T}_{V}^{n}$.

than the overall $\mathcal{O}\left(\delta t_{n}^{2}\right)$ consistency of the Heun method under the CFL condition. The accuracy order of the method is expected to be at least $\mathcal{O}\left(\delta t^{2}+h^{\min (2+\alpha, k)}\right)$.

Remark 4.4 (Constants $c_{M}$ and $c_{E}$ ). The constant $c_{M}$ is user-defined, nondimensional and of order one. The constant $c_{E}$ is also user-defined but dimensional; for instance, it can be defined as

$$
c_{E}:=\mathfrak{c}_{E} \frac{D^{1-\alpha}}{|\Omega|^{-1 / 2}\left\|U^{0}\right\|_{L^{2}(\Omega)}}
$$

or $c_{E}:=\mathfrak{c}_{E} D^{1-\alpha}\left\|U^{0}\right\|_{L^{\infty}}^{-1}$, where $D:=\operatorname{diam}(\Omega)$ and $\mathfrak{c}_{E}$ is a user-defined nondimensional constant of order one; see also Remark 3.1.

4.2. Stability analysis of RK2/Heun. We establish in this section the $L^{2}$ stability of the RK2/Heun time discretization of (2.1).

Theorem 4.1 (Stability of the RK2/Heun). There is $\Lambda_{0}>0$ that depends only on the user-defined parameters $c_{M}, \mathfrak{c}_{E}$, the Lipschitz constant of the flux, and on the mesh family constants $c_{0}, c_{i}$, and there is a constant $c$ that additionally depends linearly on $T^{2(1-\alpha)}$ so that the solution to (4.1)-(4.2) satisfies the following $L^{2}$ stability estimate for all $\lambda \leq \Lambda_{0}$ :

$$
\begin{aligned}
&\left\|U^{n+1}\right\|_{L^{2}(\Omega)}^{2}+\sum_{i=0}^{n} \delta t_{n}\left(\left\|\sqrt{\nu_{1}^{i}} \nabla U^{i}\right\|_{L^{2}(\Omega)}^{2}+\left\|\sqrt{\nu_{2}^{i}} \nabla W^{i}\right\|_{L^{2}(\Omega)}^{2}\right) \\
& \leq\left\|U_{0}\right\|_{L^{2}(\Omega)}^{2}\left(1+c \lambda^{2(1+\alpha)}(\delta t / T)^{1-2 \alpha}\right), \quad \forall t_{n} \leq T
\end{aligned}
$$

where $\delta t:=\max _{i=0, \ldots, n} \delta t_{n}$. In particular, (4.1)-(4.2) is stable provided $\alpha \leq \frac{1}{2}$. 
Proof. Step 1. Choosing $V=U^{n}$ in (4.1), $V=2 W^{n}$ in (4.2), using the conservation property (4.3), and adding the two results we obtain that

$$
\begin{array}{r}
\left\|U^{n+1}\right\|_{L^{2}(\Omega)}^{2}-\left\|U^{n}\right\|_{L^{2}(\Omega)}^{2}+\delta t_{n}\left(\left\|\sqrt{\nu_{1}^{n}} \nabla U^{n}\right\|_{L^{2}(\Omega)}^{2}+\left\|\sqrt{\nu_{2}^{n}} \nabla W^{n}\right\|_{L^{2}(\Omega)}^{2}\right) \\
\leq\left\|U^{n+1}-W^{n}\right\|_{L^{2}(\Omega)}^{2}
\end{array}
$$

The rest of the proof consists of deriving a bound on the time increment $\| U^{n+1}-$ $W^{n} \|_{L^{2}(\Omega)}^{2}$. Note that this time increment is formally second-order as can be observed by constructing (4.2) $-\frac{1}{2}(4.1)$ :

$$
\begin{aligned}
\left(U^{n+1}-W^{n}, V\right)_{\Omega}=-\frac{\delta t_{n}}{2}\left(\nabla \cdot \left(\mathbf{f}\left(W^{n}\right)\right.\right. & \left.\left.-\mathbf{f}\left(U^{n}\right)\right), V\right)_{\Omega} \\
& -\frac{\delta t_{n}}{2}\left(\nu_{2}^{n} \nabla W^{n}-\nu_{1}^{n} \nabla U^{n}, \nabla V\right)_{\Omega} .
\end{aligned}
$$

Step 2. We set $Z^{n}:=W^{n}-U^{n}$ and test (4.14) with $V=U^{n+1}-W^{n}$. The first term in the right-hand side is handled as follows:

$$
\begin{aligned}
-\frac{\delta t_{n}}{2}( & \left.\nabla \cdot\left(\mathbf{f}\left(W^{n}\right)-\mathbf{f}\left(U^{n}\right)\right), V\right)_{\Omega}=-\frac{\delta t_{n}}{2}\left(\left(\mathbf{f}^{\prime}\left(W^{n}\right)-\mathbf{f}^{\prime}\left(U^{n}\right)\right) \cdot \nabla W^{n}, V\right)_{\Omega} \\
& -\frac{\delta t_{n}}{2}\left(\mathbf{f}^{\prime}\left(U^{n}\right) \cdot \nabla\left(W^{n}-U^{n}\right), V\right)_{\Omega} \\
& \leq c_{M}^{-\frac{1}{2}} \delta t_{n}^{\frac{1}{2}} \lambda^{\frac{1}{2}}\left\|\sqrt{\nu_{2}^{n}} \nabla W^{n}\right\|_{L^{2}(\Omega)}\|V\|_{L^{2}(\Omega)}+\frac{1}{2} \lambda\left\|h \nabla Z^{n}\right\|_{L^{2}(\Omega)}\|V\|_{L^{2}(\Omega)},
\end{aligned}
$$

where we used the definition of $\nu_{2}^{n}$ to deduce that

$$
\left\|\mathbf{f}^{\prime}\left(W^{n}\right)-\mathbf{f}^{\prime}\left(U^{n}\right)\right\|_{L^{\infty}(K)}^{2} \leq\left. 4 \nu_{2}^{n}\right|_{K} h_{K}^{-1} c_{M}^{-1} \max \left(\left\|\mathbf{f}^{\prime}\left(U^{n}\right)\right\|_{L^{\infty}(K)},\left\|\mathbf{f}^{\prime}\left(W^{n}\right)\right\|_{L^{\infty}(K)}\right) .
$$

The second term in (4.14) is estimated as follows:

$$
\begin{aligned}
& -\frac{\delta t_{n}}{2}\left(\nu_{2}^{n} \nabla W^{n}-\nu_{1}^{n} \nabla U^{n}, \nabla V\right)_{\Omega} \\
& \quad \leq \frac{c_{i}}{2} \delta t_{n}^{\frac{1}{2}} \lambda^{\frac{1}{2}} c_{M}^{\frac{1}{2}}\left(\left\|\sqrt{\nu_{2}^{n}} \nabla W^{n}\right\|_{L^{2}}+\left\|\sqrt{\nu_{1}^{n}} \nabla U^{n}\right\|_{L^{2}}\right)\|V\|_{L^{2} .}
\end{aligned}
$$

Combining the above estimates gives

$$
\begin{aligned}
\left\|U^{n+1}-W^{n}\right\|_{L^{2}}^{2} & \leq \lambda^{2}\left\|h \nabla Z^{n}\right\|_{L^{2}}^{2} \\
& +\left(c_{i}^{2} c_{M}+4 c_{M}^{-1}\right) \lambda \delta t_{n}\left(\left\|\sqrt{\nu_{2}^{n}} \nabla W^{n}\right\|_{L^{2}}^{2}+\left\|\sqrt{\nu_{1}^{n}} \nabla U^{n}\right\|_{L^{2}}^{2}\right) .
\end{aligned}
$$

The two viscous terms in the right-hand side can be absorbed in the left-hand side of (4.13) provided $\Lambda_{0}$ is small enough. The remaining term $\left\|h \nabla Z^{n}\right\|_{L^{2}}$ is critical. To control this term we borrow an argument from [5] and adapt it to make it work for any polynomial degree (see Remark 4.5). The argument is based on the properties

$$
\begin{gathered}
\left\|h \nabla Z^{n}\right\|_{L^{2}(K)} \leq c_{i}\left\|Z^{n}-\Pi^{0} Z^{n}\right\|_{L^{2}(K)}, \quad \forall K \in \mathcal{T}, \\
\int_{K} \Pi^{0}\left(\nabla \cdot \mathbf{f}\left(U^{n}\right)\right)\left(Z^{n}-\Pi^{0} Z^{n}\right) \mathrm{d} \mathbf{x}=0, \quad \forall K \in \mathcal{T},
\end{gathered}
$$

where $\Pi^{0}$ is the $L^{2}$-projection onto piecewise constants, i.e., $\Pi^{0} v$ is defined on each mesh cell by $\left.\Pi^{0} v\right|_{K}=|K|^{-1} \int_{K} v \mathrm{~d} \mathbf{x}$, for all $v \in L^{2}(\Omega)$. Using inequality (4.16) in 
(4.15) implies that

$$
\begin{aligned}
\| U^{n+1}- & W^{n}\left\|_{L^{2}}^{2} \leq c_{i}^{2} \lambda^{2}\right\| Z^{n}-\Pi^{0} Z^{n} \|_{L^{2}}^{2} \\
& +\left(c_{i}^{2} c_{M}+4 c_{M}^{-1}\right) \lambda \delta t_{n}\left(\left\|\sqrt{\nu_{2}^{n}} \nabla W^{n}\right\|_{L^{2}(\Omega)}^{2}+\left\|\sqrt{\nu_{1}^{n}} \nabla U^{n}\right\|_{L^{2}(\Omega)}^{2}\right) .
\end{aligned}
$$

Step 3. We now focus our attention on the first term in the right-hand side of (4.18) and we denote $X^{n}:=Z^{n}-\Pi^{0} Z^{n}$. The defining properties of $\Pi^{0}$ and $\Pi$ imply

$$
\lambda^{2}\left\|X^{n}\right\|_{L^{2}}^{2}=\lambda^{2}\left(X^{n}, Z^{n}\right)_{\Omega}=\lambda^{2}\left(\Pi X^{n}, Z^{n}\right)_{\Omega} .
$$

Note that from (4.1) we have

$$
\left(Z^{n}, V\right)_{\Omega}=-\delta t_{n}\left(\nabla \cdot \mathbf{f}\left(U^{n}\right), V\right)_{\Omega}-\delta t_{n}\left(\nu_{1}^{n} \nabla U^{n}, \nabla V\right)_{\Omega}, \quad \forall V \in \mathbb{V}(\mathcal{T})
$$

Hence, by choosing the test function $V=\Pi X^{n}$ in this equation, we obtain

$$
\begin{aligned}
\lambda^{2}\left\|X^{n}\right\|_{L^{2}}^{2} & =\lambda^{2}\left(\Pi X^{n}, Z^{n}\right)_{\Omega} \\
& =-\lambda^{2} \delta t_{n}\left(\nabla \cdot \mathbf{f}\left(U^{n}\right), \Pi X^{n}\right)_{\Omega}-\delta t_{n} \lambda^{2}\left(\nu_{1}^{n} \nabla U^{n}, \nabla \Pi X^{n}\right)_{\Omega} .
\end{aligned}
$$

The $L^{2}$-stability of $\Pi$ and the boundedness of $\nu_{1}^{n}$ imply that the last term above can be bounded as follows:

$$
\begin{aligned}
-\delta t_{n} \lambda^{2}\left(\nu_{1}^{n} \nabla U^{n}, \nabla \Pi X^{n}\right)_{\Omega} & \leq \delta t_{n} \lambda^{2}\left\|\sqrt{\nu_{1}^{n}} \nabla U^{n}\right\|_{L^{2}(\Omega)}\left\|\sqrt{\nu_{1}^{n}} \nabla \Pi X^{n}\right\|_{L^{2}} \\
& \leq c_{i} c_{M}^{\frac{1}{2}} \delta t_{n}^{\frac{1}{2}} \lambda^{\frac{5}{2}}\left\|\sqrt{\nu_{1}^{n}} \nabla U^{n}\right\|_{L^{2}(\Omega)}\left\|\Pi X^{n}\right\|_{L^{2}} \\
& \leq c_{i} c_{M}^{\frac{1}{2}} \delta t_{n}^{\frac{1}{2}} \lambda^{\frac{5}{2}}\left\|\sqrt{\nu_{1}^{n}} \nabla U^{n}\right\|_{L^{2}(\Omega)}\left\|X^{n}\right\|_{L^{2} .}
\end{aligned}
$$

Gathering the above estimates, we can recast (4.19) into

$$
\lambda^{2}\left(1-\frac{c_{i}^{2} c_{M}}{2} \lambda^{2}\right)\left\|X^{n}\right\|_{L^{2}}^{2} \leq \frac{1}{2} \delta t_{n} \lambda\left\|\sqrt{\nu_{1}^{n}} \nabla U^{n}\right\|_{L^{2}(\Omega)}^{2}-\lambda^{2} \delta t_{n}\left(\nabla \cdot \mathbf{f}\left(U^{n}\right), \Pi X^{n}\right)_{\Omega} .
$$

If $\Lambda_{0}$ is chosen so that $\Lambda_{0} \leq c_{i}^{-1} c_{M}^{-\frac{1}{2}}$, then for all $\lambda \leq \Lambda_{0}$,

$$
\lambda^{2}\left\|X^{n}\right\|_{L^{2}}^{2} \leq \delta t_{n} \lambda\left\|\sqrt{\nu_{1}^{n}} \nabla U^{n}\right\|_{L^{2}(\Omega)}^{2}-2 \lambda^{2} \delta t_{n}\left(\nabla \cdot \mathbf{f}\left(U^{n}\right), \Pi X^{n}\right)_{\Omega} .
$$

The last term in the right-hand side of the above expression is the most complicated to estimate, and this is done by invoking the decomposition $\mathcal{T}=\mathcal{T}_{V}^{n} \cup \mathcal{T}_{S}^{n}$.

Step 4 (Control over $\mathcal{T}_{V}^{n}$ ). We use the fact that $\left.\nu_{1}^{n}\right|_{K}=c_{M} h_{K}\left\|\mathbf{f}^{\prime}\left(U^{n}\right)\right\|_{L^{\infty}(K)}$ over $\mathcal{T}_{V}^{n}$ and the $L^{2}$-stability of $\Pi$ to obtain

$$
-2 \lambda^{2} \delta t_{n}\left(\mathbf{f}^{\prime}\left(U^{n}\right) \cdot \nabla U^{n}, \Pi X^{n}\right)_{\mathcal{T}_{V}^{n}} \leq \lambda \delta t_{n}\left\|\sqrt{\nu_{1}^{n}} \nabla U^{n}\right\|_{L^{2}(\Omega)}^{2}+c_{M}^{-1} \lambda^{4}\left\|X^{n}\right\|_{L^{2}(\Omega)}^{2} .
$$

Step 5 (Control over $\left.\mathcal{T}_{S}^{n}\right)$. We handle the term $I:=-2 \lambda^{2} \delta t_{n}\left(\nabla \cdot \mathbf{f}\left(U^{n}\right), \Pi X^{n}\right)_{\mathcal{T}_{S}^{n}}$ as follows:

$$
\frac{1}{2} I=-\lambda^{2} \delta t_{n}\left(\Pi^{0}\left(\nabla \cdot \mathbf{f}\left(U^{n}\right)\right), \Pi X^{n}\right)_{\mathcal{T}_{S}^{n}}-\lambda^{2} \delta t_{n}\left(\nabla \cdot \mathbf{f}\left(U^{n}\right)-\Pi^{0}\left(\nabla \cdot \mathbf{f}\left(U^{n}\right)\right), \Pi X^{n}\right)_{\mathcal{T}_{S}^{n}} .
$$

We now need to control $-\lambda^{2} \delta t_{n}\left(\Pi^{0} \nabla \cdot \mathbf{f}\left(U^{n}\right), \Pi X^{n}\right)_{\mathcal{T}_{S}^{n}}$; the key to the whole proof is here. Let us first recall that $X^{n}:=Z^{n}-\Pi^{0} Z^{n}$ and (4.17) holds since $\Pi^{0} \nabla \cdot \mathbf{f}\left(U^{n}\right)$ is piecewise constant; this property in turn implies that

$$
-\lambda^{2} \delta t_{n}\left(\Pi^{0} \nabla \cdot \mathbf{f}\left(U^{n}\right), \Pi X^{n}\right)_{\mathcal{T}_{S}^{n}}=-\lambda^{2} \delta t_{n}\left(\Pi^{0} \nabla \cdot \mathbf{f}\left(U^{n}\right), \Pi X^{n}-X^{n}\right)_{\mathcal{T}_{S}^{n}} .
$$


It is at this point that we use the fact that we are testing with $\Pi X^{n}-X^{n}$. In particular, we are going to use the key property

$$
\left(\mathcal{R}_{\mathcal{T}_{S}^{n}}\left(U^{n}-U^{n-1}\right), \Pi X^{n}-X^{n}\right)_{\mathcal{T}_{S}^{n}}=0,
$$

where the restriction operator $\mathcal{R}_{\mathcal{T}_{S}^{n}}$ is defined in (2.13). The above orthogonality property allows us to construct a residual $R^{n}:=\delta t_{n-1}^{-1}\left(U^{n}-U^{n-1}\right)+\nabla \cdot \mathbf{f}\left(U^{n}\right)$ so that

$$
\begin{aligned}
\frac{1}{2} I=- & \lambda^{2} \delta t_{n}\left(\Pi^{0}\left(\nabla \cdot \mathbf{f}\left(U^{n}\right)\right)+\delta t_{n-1}^{-1} \mathcal{R}_{\mathcal{T}_{S}^{n}}\left(U^{n}-U^{n-1}\right), \Pi X^{n}-X^{n}\right)_{\mathcal{T}_{S}^{n}} \\
& -\lambda^{2} \delta t_{n}\left(\left(\nabla \cdot \mathbf{f}\left(U^{n}\right)-\Pi^{0}\left(\nabla \cdot \mathbf{f}\left(U^{n}\right)\right), \Pi X^{n}\right)_{\mathcal{T}_{S}^{n}}\right. \\
=- & \lambda^{2} \delta t_{n}\left(R^{n}, \Pi X^{n}-X^{n}\right)_{\dot{\mathcal{T}}_{S}^{n}}-\lambda^{2} \delta t_{n}\left(\left(\Pi^{0}\left(\nabla \cdot \mathbf{f}\left(U^{n}\right)\right)-\nabla \cdot \mathbf{f}\left(U^{n}\right), \Pi X^{n}-X^{n}\right)_{\dot{\mathcal{T}}_{S}^{n}}\right. \\
& -\lambda^{2} \delta t_{n}\left(\Pi^{0}\left(\nabla \cdot \mathbf{f}\left(U^{n}\right)\right)+\delta t_{n-1}^{-1} \mathcal{R}_{\mathcal{T}_{S}^{n}}\left(U^{n}-U^{n-1}\right), \Pi X^{n}-X^{n}\right)_{\mathcal{L}^{n}} \\
& -\lambda^{2} \delta t_{n}\left(\left(\nabla \cdot \mathbf{f}\left(U^{n}\right)-\Pi^{0}\left(\nabla \cdot \mathbf{f}\left(U^{n}\right)\right), \Pi X^{n}\right)_{\mathcal{T}_{S}^{n}},\right.
\end{aligned}
$$

where $\mathcal{L}^{n}$ is the layer of elements in $\mathcal{T}_{S}^{n}$ that is between $\dot{\mathcal{T}}_{S}^{n}$ and $\mathcal{T}_{V}^{n}$, i.e., $\dot{\mathcal{T}}_{S}^{n} \cup \mathcal{L}^{n}=$ $\mathcal{T}_{S}^{n}$. We reorganize the above identity as follows:

$$
\begin{gathered}
\frac{1}{2} I=-\lambda^{2} \delta t_{n}\left(R^{n}, \Pi X^{n}-X^{n}\right)_{\dot{\mathcal{T}}_{S}^{n}}+\lambda^{2} \delta t_{n}\left(\left(\Pi^{0}\left(\nabla \cdot \mathbf{f}\left(U^{n}\right)\right)-\nabla \cdot \mathbf{f}\left(U^{n}\right), X^{n}\right)_{\mathcal{T}_{S}^{n}}\right. \\
-\lambda^{2} \delta t_{n}\left(\nabla \cdot \mathbf{f}\left(U^{n}\right)+\delta t_{n-1}^{-1} \mathcal{R}_{\mathcal{T}_{S}^{n}}\left(U^{n}-U^{n-1}\right), \Pi X^{n}-X^{n}\right)_{\mathcal{L}^{n}} .
\end{gathered}
$$

Let us denote $I_{1}, I_{2}$ and $I_{3}$ the three terms in the right-hand side. We know that $c_{E} h_{K}^{\alpha}\left\|R^{n}\right\|_{L^{\infty}(K)} \leq c_{M}\left\|\mathbf{f}^{\prime}\left(U^{n}\right)\right\|_{L^{\infty}(K)}$, for all $K \in \mathcal{T}_{S}$; this implies that

$$
\begin{aligned}
I_{1}:= & -\lambda^{2} \delta t_{n}\left(R^{n}, \Pi X^{n}-X^{n}\right)_{\dot{\mathcal{T}}_{S}^{n}} \leq 2 \lambda^{2} \delta t_{n}\left\|R^{n}\right\|_{L^{2}\left(\dot{\mathcal{T}}_{S}^{n}\right)}\left\|X^{n}\right\|_{L^{2}(\Omega)} \\
& \leq \epsilon \lambda^{2}\left\|X^{n}\right\|_{L^{2}(\Omega)}^{2}+\frac{c_{M}^{2}}{c_{E}^{2} \epsilon} \lambda^{2(1+\alpha)} \delta t_{n}^{2(1-\alpha)}\left\|\mathbf{f}^{\prime}\right\|_{L^{\infty}(\Omega)}^{2(1-\alpha)}|\Omega| \\
& \leq \epsilon \lambda^{2}\left\|X^{n}\right\|_{L^{2}(\Omega)}^{2}+\frac{c_{M}^{2} g_{E}}{\epsilon} \lambda^{2(1+\alpha)} \delta t_{n}^{2(1-\alpha)}\left\|U^{0}\right\|_{L^{2}(\Omega)}^{2},
\end{aligned}
$$

where we set

$$
g_{E}:=\left\|\mathbf{f}^{\prime}\right\|_{L^{\infty}(\mathbb{R})}^{2(1-\alpha)}|\Omega| c_{E}^{-2}\left\|U^{0}\right\|_{L^{2}(\Omega)}^{-2},
$$

and $\epsilon>0$ is a constant yet to be chosen. To control $I_{2}$, we first observe that if $\left.\nabla U^{n}\right|_{K}=0$ or $\left.\mathbf{f}^{\prime}\left(U^{n}\right)\right|_{K}=0$, then $\delta t_{n}\left\|\nabla \cdot \mathbf{f}\left(U^{n}\right)-\Pi^{0}\left(\nabla \cdot \mathbf{f}\left(U^{n}\right)\right)\right\|_{L^{\infty}(K)}^{2}=0$, otherwise,

$$
\begin{aligned}
\delta t_{n}\left\|\nabla \cdot \mathbf{f}\left(U^{n}\right)-\Pi^{0}\left(\nabla \cdot \mathbf{f}\left(U^{n}\right)\right)\right\|_{L^{\infty}(K)}^{2} & \leq \lambda h_{K} 4 \operatorname{losc}_{K}\left(\mathbf{f}, U^{n}\right)\left\|\nabla U^{n}\right\|_{L^{\infty}(K)}^{2} \\
& \leq\left. 4 \frac{\lambda}{c_{M}} \nu_{1}^{n}\right|_{K}\left\|\nabla U^{n}\right\|_{L^{\infty}(K)}^{2} .
\end{aligned}
$$

Since the mesh is affine (2.9) also holds for $\nabla U^{n}$, i.e.,

$$
\left\|\nabla U^{n}\right\|_{L^{\infty}(K)}^{2} \leq c_{\infty}^{2}|K|^{-1}\left\|\nabla U^{n}\right\|_{L^{2}(K)}^{2} .
$$


Upon using this inequality and the $L^{2}$-stability of $\Pi$ we infer that

$$
\begin{aligned}
I_{2}:= & -\lambda^{2} \delta t_{n}\left(\nabla \cdot \mathbf{f}\left(U^{n}\right)-\Pi^{0}\left(\nabla \cdot \mathbf{f}\left(U^{n}\right)\right), \Pi X^{n}-X^{n}\right)_{\mathcal{T}_{S}^{n}} \\
\leq & 4 c_{\infty} c_{M}^{-\frac{1}{2}} \lambda^{\frac{5}{2}} \delta t_{n}^{\frac{1}{2}}\left\|\sqrt{\nu_{1}^{n}} \nabla U^{n}\right\|_{L^{2}(\Omega)}\left\|X^{n}\right\|_{L^{2}(\Omega)} \leq \epsilon \lambda^{2}\left\|X^{n}\right\|_{L^{2}(\Omega)}^{2} \\
& +\frac{4 c_{\infty}^{2}}{\epsilon c_{M}} \lambda^{3} \delta t_{n}\left\|\sqrt{\nu_{1}^{n}} \nabla U^{n}\right\|_{L^{2}(\Omega)}^{2} .
\end{aligned}
$$

We proceed as follows to control $I_{3}$,

$$
\begin{aligned}
I_{3}:= & -\lambda^{2} \delta t_{n}\left(\nabla \cdot \mathbf{f}\left(U^{n}\right)+\delta t_{n-1}^{-1} \mathcal{R}_{\mathcal{T}_{S}^{n}}\left(U^{n}-U^{n-1}\right), \Pi X^{n}-X^{n}\right)_{\mathcal{L}^{n}} \\
\leq & \lambda^{2} \delta t_{n}\left(\left\|\nabla \cdot \mathbf{f}\left(U^{n}\right)\right\|_{L^{2}\left(\mathcal{L}^{n}\right)}+c_{\mathcal{R}}\left\|\delta t_{n-1}^{-1}\left(U^{n}-U^{n-1}\right)\right\|_{L^{2}\left(\mathcal{L}^{n}\right)}\right)\left\|\Pi X^{n}-X^{n}\right\|_{L^{2}\left(\mathcal{L}^{n}\right)} \\
\leq & \lambda^{2} \delta t_{n}\left(\left\|\nabla \cdot \mathbf{f}\left(U^{n}\right)\right\|_{L^{2}\left(\mathcal{L}^{n}\right)}+c_{\mathcal{R}}\left\|\nabla \cdot \mathbf{f}\left(U^{n}\right)\right\|_{L^{2}\left(\mathcal{L}^{n}\right)}\right. \\
& \left.+c_{\mathcal{R}}\left\|R^{n}\right\|_{L^{2}\left(\mathcal{L}^{n}\right)}\right)\left\|\Pi X^{n}-X^{n}\right\|_{L^{2}(\Omega)} \\
\leq & 2\left(1+c_{\mathcal{R}}\right) c_{M}^{-\frac{1}{2}} \lambda^{\frac{5}{2}} \delta t_{n}^{\frac{1}{2}}\left\|\sqrt{\nu_{1}^{n}} \nabla U^{n}\right\|_{L^{2}(\Omega)}\left\|X^{n}\right\|_{L^{2}(\Omega)} \\
& +2 c_{\mathcal{R}} \lambda^{2} \delta t_{n}\left\|R^{n}\right\|_{L^{2}\left(\mathcal{L}^{n}\right)}\left\|X^{n}\right\|_{L^{2}(\Omega)},
\end{aligned}
$$

where we used that $\left.\nu_{1}^{n}\right|_{K}=c_{M} h_{k}\left\|\mathbf{f}^{\prime}\left(U^{n}\right)\right\|_{L^{\infty}(K)}$ for all $K \in \mathcal{L}^{n}$ together with the $L^{2}$-stability of $\Pi^{0}, \Pi$ and $\mathcal{R}_{\mathcal{T}_{S}^{n}}$ (Lemma 2.1). Using again that $c_{E} h_{K}^{\alpha}\left\|R^{n}\right\|_{L^{\infty}(K)} \leq$ $c_{M}\left\|\mathbf{f}^{\prime}\left(U^{n}\right)\right\|_{L^{\infty}(K)}$ for all $K \in \mathcal{L}^{n} \subset \mathcal{T}_{S}^{n}$ we infer that

$$
I_{3} \leq \epsilon \lambda^{2}\left\|X^{n}\right\|_{L^{2}}+\frac{c_{\mathcal{R}}^{2} c_{M}^{2} g_{E}}{\epsilon} \lambda^{2(1+\alpha)} \delta t_{n}^{2(1-\alpha)}\left\|U^{0}\right\|_{L^{2}}^{2}+\frac{c^{\prime}}{\epsilon c_{M}} \lambda^{3} \delta t_{n}\left\|\sqrt{\nu_{1}^{n}} \nabla U^{n}\right\|_{L^{2}}^{2},
$$

where $g_{E}$ is given by (4.22). Gathering the estimates on $I_{1}, I_{2}$, and $I_{3}$ we finally deduce the following estimate:

$$
\begin{aligned}
& -2 \lambda^{2} \delta t_{n}\left(\mathbf{f}^{\prime}\left(U^{n}\right) \cdot \nabla U^{n}, \Pi X^{n}\right)_{\Omega} \leq 6 \epsilon \lambda^{2}\left\|X^{n}\right\|_{L^{2}} \\
& \quad+\frac{c c_{M}^{2} g_{E}}{\epsilon} \lambda^{2(1+\alpha)} \delta t_{n}^{2(1-\alpha)}\left\|U^{0}\right\|_{L^{2}(\Omega)}^{2}+\frac{c^{\prime}}{\epsilon c_{M}} \lambda^{3} \delta t_{n}\left\|\sqrt{\nu_{1}^{n}} \nabla U^{n}\right\|_{L^{2}(\Omega)}^{2}
\end{aligned}
$$

Step 6 (Conclusion). Combining (4.21) and (4.23), and setting $\epsilon=\frac{1}{12}$ we can finally rewrite (4.20) as follows:

$$
\begin{aligned}
\lambda^{2}\left\|X^{n}\right\|_{L^{2}(\Omega)} \leq & c c_{M}^{2} g_{E} \lambda^{2(1+\alpha)} \delta t_{n}^{2(1-\alpha)}\left\|U^{0}\right\|_{L^{2}(\Omega)}^{2} \\
& +c^{\prime} \lambda\left(1+\lambda^{2} c_{M}^{-1}\right) \delta t_{n}\left\|\sqrt{\nu_{1}^{n}} \nabla U^{n}\right\|_{L^{2}(\Omega)}^{2} .
\end{aligned}
$$

We now combine the above estimate with (4.18) to obtain

$$
\begin{aligned}
\| U^{n+1}- & W^{n}\left\|_{L^{2}}^{2} \leq c c_{M}^{2} g_{E} \lambda^{2(1+\alpha)} \delta t_{n}^{2(1-\alpha)}\right\| U^{0} \|_{L^{2}}^{2} \\
& +c^{\prime}\left(1+c_{M} c_{i}^{2}+\left(4+\lambda^{2}\right) c_{M}^{-1}\right) \lambda \delta t_{n}\left(\left\|\sqrt{\nu_{1}^{n}} \nabla U^{n}\right\|_{L^{2}}^{2}+\left\|\sqrt{\nu_{2}^{n}} \nabla W^{n}\right\|_{L^{2}}^{2}\right) .
\end{aligned}
$$

Provided $\Lambda_{0}$ is chosen so that $c^{\prime} \Lambda_{0}\left(1+c_{M} c_{i}^{2}+\left(4+\Lambda_{0}^{2}\right) c_{M}^{-1}\right) \leq \frac{1}{2}$, the above bound together with (4.13) implies that the following energy estimate holds for $\lambda \leq \Lambda_{0}$,

$$
\begin{aligned}
\left\|U^{n+1}\right\|_{L^{2}}^{2}-\left\|U^{n}\right\|_{L^{2}}^{2}+\delta t_{n}\left(\left\|\sqrt{\nu_{1}^{n}} \nabla U^{n}\right\|_{L^{2}}^{2}\right. & \left.+\left\|\sqrt{\nu_{2}^{n}} \nabla W^{n}\right\|_{L^{2}}^{2}\right) \\
& \leq c c_{M}^{2} g_{E} \lambda^{2(1+\alpha)} \delta t_{n}^{2(1-\alpha)}\left\|U^{0}\right\|_{L^{2}(\Omega)}^{2}
\end{aligned}
$$


Summing this inequality from $n=0$ to $N$ gives

$$
\begin{aligned}
\left\|U^{n+1}\right\|_{L^{2}}^{2}+\sum_{i=0}^{n} \delta t_{n}\left(\left\|\sqrt{\nu_{1}^{i}} \nabla U^{i}\right\|_{L^{2}}^{2}+\left\|\sqrt{\nu_{2}^{i}} \nabla W^{i}\right\|_{L^{2}}^{2}\right) & \\
& \leq\left\|U_{0}\right\|_{L^{2}}^{2}\left(1+c c_{M}^{2} g_{E} T^{2(1-\alpha)} \lambda^{2(1+\alpha)}(\delta t / T)^{1-2 \alpha}\right)
\end{aligned}
$$

which is the desired estimate. Note that $g_{E} T^{2(1-\alpha)}$ is a dimensionless constant.

Remark 4.5 (No restriction on the polynomial order). We emphasize that one of the key steps in the above stability proof is the control of the term $\left\|h \nabla Z^{n}\right\|_{L^{2}(K)}$. The two key arguments consist of the following: (i) subtracting the projection onto constants of $Z^{n}$, i.e., $\left\|h \nabla\left(Z^{n}-\Pi^{0} Z^{n}\right)\right\|_{L^{2}(K)}$, so as to be able to use the inverse estimate (4.16); (ii) forming a residual relying on the orthogonality property (4.17). This argument is borrowed from [5], where it was restricted to piecewise linear finite elements. We have extended it to any polynomial degree $k \geq 1$ by taking advantage of the nonlinear viscosity $\nu_{1}^{n}$ which satisfies

$$
c_{M} h_{K} \operatorname{Osc}_{K}\left(\mathbf{f}, U^{n}\right) \leq\left.\nu_{1}^{n}\right|_{K}, \quad \forall K \in \dot{\mathcal{T}_{S}^{n}} .
$$

Remark 4.6 (Restriction on $\alpha$ ). The restriction $\alpha<\frac{1}{2}$ for stability in Theorem 4.1 seems to be purely technical. Thorough numerical experiments have shown that the method is stable and convergent with $\alpha=1$. We then conjecture that Theorem 4.1 should hold in the range $\alpha \in[0,1]$.

\section{Midpoint RK2}

The algorithm presented in 4.1 relies on a viscosity that is built from the previous time step (see (4.5)-(4.7)). This may seem a little odd since we are solving a Cauchy problem. We propose in this section an alternative technique that consists of constructing the viscosity on the fly. The method is implemented with the midpoint RK2 technique.

5.1. The algorithm. Let $t_{0}=0$ and let $U^{0} \in \mathbb{V}(\mathcal{T})$ be an approximation of $u_{0}$. Let $\lambda>0$ be a CFL number. Let $U^{n} \in \mathbb{V}(\mathcal{T})$ be the approximation of $u$ at time $t_{n}$, $n \geq 0$. Let $\delta t_{n}$ be a given time step possibly restricted later by the CFL number (see (5.3) ) and set $t_{n+1}=t_{n}+\delta t_{n}$. The midpoint RK2 algorithm is formulated as follows: Seek $W^{n} \in \mathbb{V}(\mathcal{T})$ and $U^{n+1} \in \mathbb{V}(\mathcal{T})$ satisfying

$$
\begin{aligned}
& \left(W^{n}, V\right)_{\Omega}-\left(U^{n}, V\right)_{\Omega}+\frac{\delta t_{n}}{2}\left(\nabla \cdot \mathbf{f}\left(U^{n}\right), V\right)_{\Omega}=0, \\
& \left(U^{n+1}, V\right)_{\Omega}-\left(U^{n}, V\right)_{\Omega}+\delta t_{n}\left(\nabla \cdot \mathbf{f}\left(W^{n}\right), V\right)_{\Omega}+\delta t_{n}\left(\nu^{n} \nabla W^{n}, \nabla V\right)_{\Omega}=0,
\end{aligned}
$$

for all $V \in \mathbb{V}(\mathcal{T})$, where the viscosity $\nu^{n}$ is defined below. We assume that the time step satisfies the condition

$$
\delta t_{n} \leq \lambda \min _{K \in \mathcal{T}} \frac{h_{K}}{\max \left(\left\|\mathbf{f}^{\prime}\left(U^{n}\right)\right\|_{L^{\infty}(K)},\left\|\mathbf{f}^{\prime}\left(W^{n}\right)\right\|_{L^{\infty}(K)}\right)} .
$$

Note that the above condition can only be verified a posteriori. If the condition (5.3) is not satisfied, the computation of $W^{n}$ and $U^{n+1}$ is redone with a smaller time step, say $\delta t_{n}$ is divided by 1.5. This procedure always terminates due to the uniform Lipschitz assumption on the flux f; i.e., picking $\delta t_{n}$ smaller than $\frac{1}{\left\|\mathbf{f}^{\prime}\right\|_{L^{\infty}(\mathbb{R})}} \min _{K \in \mathcal{T}} h_{K}$ 
always guarantees that (5.3) holds. To avoid issues induced by the boundary condition we assume that $W^{n}$ satisfy the following conservation properties:

$$
\left(\nabla \cdot \mathbf{f}\left(W^{n}\right), W^{n}\right)_{\Omega} \geq 0 .
$$

We refer to $\$ 2.1$ for a discussion on the validity of this assumption.

Let $c_{M}>0, c_{E}>0$ and $\alpha \geq 0$ be three real numbers, and we introduce the following time-dependent partition of $\mathcal{T}=\mathcal{T}_{V}^{n} \cup \mathcal{T}_{S}^{n}, \mathcal{L}^{n}:=\mathcal{T}_{S}^{n} \backslash \dot{\mathcal{T}_{S}^{n}}$,

$$
\mathcal{T}_{S}^{n}:=\left\{K \in \mathcal{T}: c_{E}\left\|h^{\alpha} R^{n}\right\|_{L^{\infty}(K)} \leq c_{M}\left\|\mathbf{f}^{\prime}\left(U^{n}\right)\right\|_{L^{\infty}(K)}\right\}, \quad \mathcal{T}_{V}^{n}:=\mathcal{T} \backslash \mathcal{T}_{S}^{n},
$$

where the residual $R^{n}$ is defined by

$$
R^{n}:=2 \frac{W^{n}-U^{n}}{\delta t_{n}}+\nabla \cdot \mathbf{f}\left(W^{n}\right) .
$$

We define the viscosity $\nu^{n}: \Omega \rightarrow \mathbb{R}$ at time $t_{n}, n \geq 1$, as

$$
\left.\nu^{n}\right|_{K}= \begin{cases}\min \left(\left.\nu_{M}^{n}\right|_{K},\left.\nu_{1}^{n}\right|_{K}\right), & \text { if } K \in \mathcal{T}_{V}^{n} \cup \dot{\mathcal{T}}_{S}^{n}, \\ \left.\nu_{M}^{n}\right|_{K} & \text { if } K \in \mathcal{L}^{n},\end{cases}
$$

where

$$
\begin{aligned}
\left.\nu_{M}^{n}\right|_{K} & :=c_{M} h_{K} \max \left(\left\|\mathbf{f}^{\prime}\left(U^{n}\right)\right\|_{L^{\infty}(K)},\left\|\mathbf{f}^{\prime}\left(W^{n}\right)\right\|_{L^{\infty}(K)}\right), \\
\left.\nu_{1}^{n}\right|_{K} & :=h_{K} \max \left(c_{E}\left\|h^{\alpha} R^{n}\right\|_{L^{\infty}(K)}, c_{M} \operatorname{Osc}_{K}\left(\mathbf{f}, W^{n}\right), c_{M} \mathrm{nl}_{K}\left(\mathbf{f}, W^{n}, U^{n}\right)\right) .
\end{aligned}
$$

The oscillation $\operatorname{osc}_{K}\left(\mathbf{f}, W^{n}\right)$ is defined in (4.8) and the nonlinear variation $\mathrm{nl}_{K}\left(\mathbf{f}, W^{n}, U^{n}\right)$ is defined in (4.10).

Remark 5.1 (Consistency of viscosities). The set $\mathcal{T}_{V}^{n} \cup \mathcal{L}^{n}$ is composed of the elements where the viscosity saturates to first-order, $\nu^{n}=c_{M} \max \left(\left\|h \mathbf{f}^{\prime}\left(U^{n}\right)\right\|_{L^{\infty}(K)}\right.$, $\left.\left\|h \mathbf{f}^{\prime}\left(W^{n}\right)\right\|_{L^{\infty}(K)}\right)$, and $\mathcal{T}_{S}^{n}$ is composed of the elements where the viscosity is formally higher-order, $\nu^{n} \approx c_{E}\left\|h^{1+\alpha} R^{n}\right\|_{L^{\infty}(K)}$. Note that $\left\|h^{1+\alpha} R^{n}\right\|_{L^{\infty}(K)}$ is formally of order $\mathcal{O}\left(h_{K}^{1+\alpha} \delta t_{n}\right)$, whereas $h_{K} \operatorname{Osc}_{K}\left(\mathbf{f}, W^{n}\right)$ and $h_{K} \mathrm{nl}_{K}\left(\mathbf{f}, W^{n}, U^{n}\right)$ are of order $\mathcal{O}\left(h_{K}^{3}\right)$ and $\mathcal{O}\left(h_{K} \delta t_{n}^{2}\right)$, respectively. We refer to Remarks 4.14 .3 for discussions on the viscosities. Note again that the consistency error induced by the entropy viscosity is of higher order than that of the second-order RK2 method.

Remark 5.2 (Definition of $c_{M}$ and $c_{E}$ ). The constants $c_{M}$ and $c_{E}$ are user-defined; $c_{M}$ is nondimensional and of order one, whereas $c_{E}$ is dimensional. For instance, just like in Remark 4.4 one can set

$$
c_{E}:=\mathfrak{c}_{E} \frac{D^{1-\alpha}}{|\Omega|^{-1 / 2}\left\|U^{0}\right\|_{L^{2}(\Omega)}}
$$

or $c_{E}:=\mathfrak{c}_{E} D^{1-\alpha}\left\|u_{0}\right\|_{L^{\infty}(\Omega)}^{-1}$, where $D:=\operatorname{diam}(\Omega)$ and $\mathfrak{c}_{E}$ is a user-defined nondimensional constant of order one; see also Remark 3.1 .

We mention two useful bounds that we will use repeatedly. On one hand,

$$
\delta t_{n}\left\|\mathbf{f}^{\prime}(V) \cdot \nabla \varphi\right\|_{L^{2}(\tau)}^{2} \leq c_{M}^{-1} \lambda\left\|\sqrt{\nu^{n}} \nabla \varphi\right\|_{L^{2}(\tau)}^{2},
$$

holds for $V=U^{n}$ or $V=W^{n}$ and for any subset $\tau \subset \mathcal{T}_{V}^{n} \cup \mathcal{L}^{n}$ and any $\varphi \in H^{1}(\tau)$. On the other hand,

$$
\begin{aligned}
\left.\nu^{n}\right|_{K} \leq c_{M} \max \left(\left\|h \mathbf{f}^{\prime}\left(U^{n}\right)\right\|_{L^{\infty}(K)},\left\|h \mathbf{f}^{\prime}\left(W^{n}\right)\right\|_{L^{\infty}(K)}\right), & \forall K \in \mathcal{T}, \\
\max \left(c_{M} h_{K} \operatorname{Osc}_{K}\left(\mathbf{f}, W^{n}\right), c_{M} h_{K} \mathrm{nl}_{K}\left(\mathbf{f}, W^{n}, U^{n}\right)\right) \leq\left.\nu^{n}\right|_{K}, & \forall K \in \mathcal{T} .
\end{aligned}
$$


5.2. Stability analysis of RK2/midpoint. We now analyze the $L^{2}$-stability of the Midpoint time discretization of (2.1).

Theorem 5.1 (Stability of RK2/midpoint). Let $\left(U^{i}\right)_{i=0}^{n+1},\left(W^{i}\right)_{i=0}^{n}$ be the sequences produced by the algorithm (5.1) -(5.2) -(5.7). There is $\Lambda_{0}>0$ that depends only on the user-defined parameters $c_{M}, \mathfrak{c}_{E}$, the Lipschitz constant of the flux, and on the mesh family constants $c_{0}, c_{i}$, and there is a constant $c$ that additionally depends linearly on $T^{2(1-\alpha)}$ so that the following $L^{2}$-stability estimate holds for all $t_{n} \leq T$ and all $\lambda \in\left(0, \Lambda_{0}\right]$,

$$
\left\|U^{n+1}\right\|_{L^{2}(\Omega)}^{2}+\sum_{i=0}^{n} \delta t_{i}\left\|\sqrt{\nu^{i}} \nabla W^{n}\right\|_{L^{2}(\Omega)}^{2} \leq\left\|U^{0}\right\|_{L^{2}(\Omega)}^{2}\left(1+c \lambda^{2(1+\alpha)}(\delta t / T)^{1-2 \alpha}\right),
$$

where $\delta t:=\max _{i=0, \ldots, n} \delta t_{i}$. Moreover, the algorithm is $L^{2}$-stable if $\alpha \leq \frac{1}{2}$.

Proof. The proof is similar to that provided in $₫$ for the Heun method and we only outline the main steps.

Step 1. Testing (5.2) with $W^{n}$ gives

$$
\left\|U^{n+1}\right\|_{L^{2}(\Omega)}^{2}-\left\|U^{n}\right\|_{L^{2}(\Omega)}^{2}+2 \delta t_{n}\left\|\sqrt{\nu^{n}} \nabla W^{n}\right\|_{L^{2}(\Omega)}^{2} \leq\left(Y^{n}, U^{n+1}-U^{n}\right)_{\Omega},
$$

where we used the conservation property (5.4) and the notation

$$
Y^{n}=U^{n+1}+U^{n}-2 W^{n} .
$$

In view of (5.14), we need to establish a bound on $\left\|Y^{n}\right\|_{L^{2}(\Omega)}$. The linear combination (5.2) $-2 \times(5.1)$ gives us a way to control $Y$ :

$$
\begin{aligned}
& \left(Y^{n}, V\right)_{\Omega}=-\delta t_{n}\left(\left(\mathbf{f}^{\prime}\left(W^{n}\right)-\mathbf{f}^{\prime}\left(U^{n}\right)\right) \cdot \nabla W^{n}, V\right)_{\Omega} \\
& \quad-\delta t_{n}\left(\mathbf{f}^{\prime}\left(U^{n}\right) \nabla\left(W^{n}-U^{n}\right), V\right)_{\Omega}-\delta t_{n}\left(\nu^{n} \nabla W^{n}, \nabla V\right)_{\Omega}, \quad \forall V \in \mathbb{V}(\mathcal{T}) .
\end{aligned}
$$

Owing to the definition of the viscosity $\nu^{n}$ (see (4.10) and (5.13)), we have

$$
\delta t_{n}\left\|\mathbf{f}^{\prime}\left(W^{n}\right)-\mathbf{f}^{\prime}\left(U^{n}\right)\right\|_{L^{\infty}(K)}^{2} \leq\left. 4 \nu^{n}\right|_{K} \lambda c_{M}^{-1},
$$

which in turn gives

$$
-\delta t_{n}\left(\left(\mathbf{f}^{\prime}\left(W^{n}\right)-\mathbf{f}^{\prime}\left(U^{n}\right)\right) \cdot \nabla W^{n}, V\right)_{\Omega} \leq 2 c_{M}^{-\frac{1}{2}} \lambda^{\frac{1}{2}} \delta t_{n}^{\frac{1}{2}}\left\|\sqrt{\nu^{n}} \nabla W^{n}\right\|_{L^{2}(\Omega)}\|V\|_{L^{2}(\Omega)} .
$$

The second term in the right-hand side of (5.15) is handled as follows:

$$
-\delta t_{n}\left(\mathbf{f}^{\prime}\left(U^{n}\right) \nabla\left(W^{n}-U^{n}\right), V\right)_{\Omega} \leq \lambda\left\|h \nabla\left(W^{n}-U^{n}\right)\right\|_{L^{2}(\Omega)}\|V\|_{L^{2}(\Omega)} .
$$

For the third term in the right-hand side of (5.15), we use the bound (5.12) on $\left.\nu^{n}\right|_{K}$ and an inverse estimate

$$
-\delta t_{n}\left(\nu^{n} \nabla W^{n}, \nabla V\right)_{\Omega} \leq c_{i} c_{M}^{\frac{1}{2}} \lambda^{\frac{1}{2}} \delta t_{n}^{\frac{1}{2}}\left\|\sqrt{\nu^{n}} \nabla W^{n}\right\|_{L^{2}(\Omega)}\|V\|_{L^{2}(\Omega)} .
$$

Gathering the above three estimates we arrive at

$$
\left\|Y^{n}\right\|_{L^{2}(\Omega)}^{2} \leq \lambda^{2}\left\|h \nabla\left(W^{n}-U^{n}\right)\right\|_{L^{2}(\Omega)}^{2}+c\left(c_{i}^{2} c_{M}+c_{M}^{-1}\right) \lambda \delta t_{n}\left\|\sqrt{\nu^{n}} \nabla W^{n}\right\|_{L^{2}(\Omega)}^{2} .
$$

Then upon introducing the notation $Z^{n}:=W^{n}-U^{n}$, we now realize that we must find a bound on $\lambda\left\|h \nabla Z^{n}\right\|_{L^{2}(\Omega)}$. 
Step 2. Recalling that $\Pi^{0}$ is the $L^{2}$-projection over constants and that $\Pi$ is the $L^{2}$-projection onto $\mathbb{V}(\mathcal{T})$, we set $X^{n}:=Z^{n}-\Pi^{0} Z^{n}$ and we observe that

$$
c_{0}^{-2}\left\|X^{n}\right\|_{L^{2}}^{2} \leq\left\|h \nabla Z^{n}\right\|_{L^{2}}^{2}=\sum_{K \in \mathcal{T}}\left\|h \nabla X^{n}\right\|_{L^{2}(K)}^{2} \leq c_{i}^{2}\left\|X^{n}\right\|_{L^{2}}^{2}=c_{i}^{2}\left(\Pi X^{n}, Z^{n}\right)_{\Omega} .
$$

Then, (5.1) together with (5.18) and the stability of the $L^{2}$-projection yields

$$
\begin{aligned}
\lambda^{2} \| h & \nabla Z^{n} \|_{L^{2}(\Omega)}^{2} \leq-\frac{1}{2} c_{i}^{2} \delta t_{n} \lambda^{2}\left(\Pi X^{n}, \nabla \cdot \mathbf{f}\left(U^{n}\right)\right)_{\Omega} \\
& \leq-\frac{1}{2} c_{i}^{2} \delta t_{n} \lambda^{2}\left(\Pi X^{n}, \mathbf{f}^{\prime}\left(U^{n}\right) \cdot \nabla W^{n}\right)_{\Omega}+\frac{1}{2} c_{i}^{2} \delta t_{n} \lambda^{2}\left(\Pi X^{n}, \mathbf{f}^{\prime}\left(U^{n}\right) \cdot \nabla Z^{n}\right)_{\Omega} \\
& \leq-\frac{1}{2} c_{i}^{2} \delta t_{n} \lambda^{2}\left(\Pi X^{n}, \mathbf{f}^{\prime}\left(U^{n}\right) \cdot \nabla W^{n}\right)_{\Omega}+\frac{1}{2} c_{i}^{2} c_{0} \lambda^{3}\left\|h \nabla Z^{n}\right\|_{L^{2}(\Omega)}^{2} .
\end{aligned}
$$

Restricting $\Lambda_{0} \leq c_{i}^{-2} c_{0}^{-1}$, we deduce that

$$
\lambda^{2}\left\|h \nabla Z^{n}\right\|_{L^{2}(\Omega)}^{2} \leq-c_{i}^{2} \delta t_{n} \lambda^{2}\left(\Pi X^{n}, \mathbf{f}^{\prime}\left(U^{n}\right) \cdot \nabla W^{n}\right)_{\Omega} .
$$

We are now going to use different techniques to deduce a bound from above on the quantity $\delta t_{n} \lambda^{2}\left|\left(\Pi X^{n}, \mathbf{f}^{\prime}\left(U^{n}\right) \cdot \nabla W^{n}\right)_{\Omega}\right|$ in the smooth and in the viscous regions.

Step 3 (Control over $\mathcal{T}_{V}$ ). Invoking (5.11) with $V=U^{n}$ and the stability of the $L^{2}$-projection, we write

$$
\delta t_{n} \lambda^{2}\left|\left(\Pi X^{n}, \mathbf{f}^{\prime}\left(U^{n}\right) \cdot \nabla W^{n}\right)_{\mathcal{T}_{V}^{n}}\right| \leq c_{M}^{-\frac{1}{2}} \delta t_{n}^{\frac{1}{2}} \lambda^{\frac{5}{2}}\left\|X^{n}\right\|_{L^{2}(\Omega)}\left\|\sqrt{\nu^{n}} \nabla W^{n}\right\|_{L^{2}\left(\mathcal{T}_{V}^{n}\right)},
$$

which, owing to (5.18), gives

$$
\begin{aligned}
\delta t_{n} \lambda^{2}\left|\left(\Pi X^{n}, \mathbf{f}^{\prime}\left(U^{n}\right) \cdot \nabla W^{n}\right)_{\mathcal{T}_{V}^{n}}\right| \leq & \epsilon \lambda^{2}\left\|h \nabla Z^{n}\right\|_{L^{2}(\Omega)}^{2} \\
& +\frac{c_{0}^{2} c_{M}^{-1}}{4 \epsilon} \lambda^{3} \delta t_{n}\left\|\sqrt{\nu^{n}} \nabla W^{n}\right\|_{L^{2}(\Omega)}^{2},
\end{aligned}
$$

where $\epsilon$ is a constant yet to be chosen.

Step 4 (Control over $\mathcal{T}_{S}$ ). We now focus our attention on the smooth region, and we use the property that the residual (5.6) is small in the smooth region. We have

$$
\begin{aligned}
\delta t_{n} \lambda^{2}\left(\Pi X^{n}, \mathbf{f}^{\prime}\left(U^{n}\right) \cdot \nabla W^{n}\right)_{\mathcal{T}_{S}^{n}}=\delta t_{n} \lambda^{2}\left(\Pi X^{n},\left(\mathbf{f}^{\prime}\left(U^{n}\right)-\mathbf{f}^{\prime}\left(W^{n}\right)\right) \cdot \nabla W^{n}\right)_{\mathcal{T}_{S}^{n}} \\
+\delta t_{n} \lambda^{2}\left(\Pi X^{n}, \nabla \cdot \mathbf{f}\left(W^{n}\right)\right)_{\mathcal{T}_{S}^{n}} .
\end{aligned}
$$

Note that the first term in the right-hand side of the above expression is directly absorbed in the viscosity using (5.16). Indeed, the stability of $\Pi$ and (5.18) imply that

$$
\begin{aligned}
\delta t_{n} \lambda^{2}\left(\Pi X^{n},\left(\mathbf{f}^{\prime}\left(U^{n}\right)\right.\right. & \left.\left.-\mathbf{f}^{\prime}\left(W^{n}\right)\right) \cdot \nabla W^{n}\right)_{\mathcal{T}_{S}^{n}} \leq c_{M}^{-\frac{1}{2}} c_{0} \lambda^{\frac{5}{2}} \delta t^{\frac{1}{2}}\left\|\sqrt{\nu^{n}} \nabla W^{n}\right\|_{L^{2}}\left\|h \nabla Z^{n}\right\|_{L^{2}} \\
& \leq \epsilon \lambda^{2}\left\|h \nabla Z^{n}\right\|_{L^{2}(\Omega)}^{2}+\frac{c_{0}^{2} c_{M}^{-1}}{4 \epsilon} \lambda^{3} \delta t_{n}\left\|\sqrt{\nu^{n}} \nabla W^{n}\right\|_{L^{2}(\Omega)}^{2},
\end{aligned}
$$

where $\epsilon>0$ is yet to be chosen. The remaining term, $\delta t_{n} \lambda^{2}\left(\Pi X^{n}, \nabla \cdot \mathbf{f}\left(W^{n}\right)\right)_{\mathcal{T}_{S}^{n}}$, is the most critical one. We start by writing

$$
\begin{aligned}
\delta t_{n} \lambda^{2}\left(\Pi X^{n}, \nabla \cdot \mathbf{f}\left(W^{n}\right)\right)_{\mathcal{T}_{S}^{n}} & =\delta t_{n} \lambda^{2}\left(\Pi X^{n}, \Pi^{0} \nabla \cdot \mathbf{f}\left(W^{n}\right)\right)_{\mathcal{T}_{S}^{n}} \\
& +\delta t_{n} \lambda^{2}\left(\Pi X^{n}, \nabla \cdot \mathbf{f}\left(W^{n}\right)-\Pi^{0} \nabla \cdot \mathbf{f}\left(W^{n}\right)\right)_{\mathcal{T}_{S}^{n}}
\end{aligned}
$$


Taking advantage of the orthogonality of $X^{n}:=Z^{n}-\Pi^{0} Z^{n}$ with respect to piecewise constants and of the orthogonality of $X^{n}-\Pi X^{n}$ with respect to elements in $\mathbb{V}(\mathcal{T})$, we infer that

$$
\begin{aligned}
& \delta t_{n} \lambda^{2}\left(\Pi X^{n}, \nabla \cdot \mathbf{f}\left(W^{n}\right)\right)_{\mathcal{T}_{S}^{n}}=\delta t_{n} \lambda^{2}\left(\Pi X^{n}-X^{n}, \Pi^{0} \nabla \cdot \mathbf{f}\left(W^{n}\right)\right)_{\mathcal{T}_{S}^{n}} \\
& \quad+\delta t_{n} \lambda^{2}\left(\Pi X^{n}, \nabla \cdot \mathbf{f}\left(W^{n}\right)-\Pi^{0} \nabla \cdot \mathbf{f}\left(W^{n}\right)\right)_{\mathcal{T}_{S}^{n}}, \\
& \quad=\delta t_{n} \lambda^{2}\left(\Pi X^{n}-X^{n},\left(\delta t_{n}\right)^{-1} \mathcal{R}_{\mathcal{T}_{S}^{n}}\left(W^{n}-U^{n}\right)+\nabla \cdot \mathbf{f}\left(W^{n}\right)\right)_{\mathcal{T}_{S}^{n}} \\
& \quad+\delta t_{n} \lambda^{2}\left(\Pi X^{n}-X^{n}, \Pi^{0} \nabla \cdot \mathbf{f}\left(W^{n}\right)-\nabla \cdot \mathbf{f}\left(W^{n}\right)\right)_{\mathcal{T}_{S}^{n}} \\
& \quad+\delta t_{n} \lambda^{2}\left(\Pi X^{n}, \nabla \cdot \mathbf{f}\left(W^{n}\right)-\Pi^{0} \nabla \cdot \mathbf{f}\left(W^{n}\right)\right)_{\mathcal{T}_{S}^{n}},
\end{aligned}
$$

where $\mathcal{R}_{\mathcal{T}_{S}^{n}}$ is defined by (2.13) and is the identity operator over $\dot{\mathcal{T}}_{S}^{n}$. The direct decomposition of the domain partition into $\mathcal{T}=\dot{\mathcal{T}}_{S}^{n} \cup \mathcal{L}^{n} \cup \mathcal{T}_{V}^{n}$ (see Figure1) yields

$$
\begin{aligned}
& \delta t_{n} \lambda^{2}\left(\Pi X^{n}, \nabla \cdot \mathbf{f}\left(W^{n}\right)\right)_{\mathcal{T}_{S}^{n}}=\delta t_{n} \lambda^{2}\left(\Pi X^{n}-X^{n}, R^{n}\right)_{\dot{\mathcal{T}}_{S}^{n}} \\
& \quad+\delta t_{n} \lambda^{2}\left(X^{n}, \nabla \cdot \mathbf{f}\left(W^{n}\right)-\Pi^{0} \nabla \cdot \mathbf{f}\left(W^{n}\right)\right)_{\mathcal{T}_{S}^{n}} \\
& \quad+\delta t_{n} \lambda^{2}\left(\Pi X^{n}-X^{n},\left(\delta t_{n}\right)^{-1} \mathcal{R}_{\mathcal{T}_{S}^{n}}\left(W^{n}-U^{n}\right)+\nabla \cdot \mathbf{f}\left(W^{n}\right)\right)_{\mathcal{L}^{n}} \\
& \quad=: I_{1}+I_{2}+I_{3} .
\end{aligned}
$$

Proceeding as in the proof of Theorem 4.1 we obtain the following bounds for each term:

$$
\begin{aligned}
& I_{1} \leq \epsilon \lambda^{2}\left\|X^{n}\right\|_{L^{2}(\Omega)}^{2}+\frac{c_{M}^{2} g_{E}}{\epsilon} \lambda^{2(1+\alpha)} \delta t_{n}^{2(1-\alpha)}\left\|U^{0}\right\|_{L^{2}(\Omega)}^{2}, \\
& I_{2} \leq \epsilon \lambda^{2}\left\|X^{n}\right\|_{L^{2}(\Omega)}^{2}+\frac{4 c_{\infty}^{2}}{\epsilon c_{M}} \lambda^{3} \delta t_{n}\left\|\sqrt{\nu^{n}} \nabla W^{n}\right\|_{L^{2}(\Omega)}^{2}, \\
& I_{3} \leq \epsilon \lambda^{2}\left\|X^{n}\right\|_{L^{2}}+\frac{c c_{M}^{2} g_{E}}{\epsilon} \lambda^{2(1+\alpha)} \delta t_{n}^{2(1-\alpha)}\left\|U^{0}\right\|_{L^{2}(\Omega)}^{2}+\frac{c^{\prime}}{\epsilon c_{M}} \lambda^{3} \delta t_{n}\left\|\sqrt{\nu^{n}} \nabla W^{n}\right\|_{L^{2}(\Omega)}^{2},
\end{aligned}
$$

where $g_{E}$ is defined in (4.22). Gathering the above estimates and using (5.18) yields

$$
\begin{aligned}
& \delta t_{n} \lambda^{2}\left(\Pi X^{n}, \nabla \cdot \mathbf{f}\left(W^{n}\right)\right)_{\mathcal{T}_{S}^{n}} \leq 3 c_{0}^{2} \epsilon \lambda^{2}\left\|h \nabla Z^{n}\right\|_{L^{2}} \\
& \quad+c \frac{c_{M}^{2} g_{E}}{\epsilon} \lambda^{2(1+\alpha)} \delta t_{n}^{2(1-\alpha)}\left\|U^{0}\right\|_{L^{2}(\Omega)}^{2}+\frac{c^{\prime}}{\epsilon c_{M}} \lambda^{3} \delta t_{n}\left\|\sqrt{\nu_{2}^{n}} \nabla U^{n}\right\|_{L^{2}(\Omega)}^{2} .
\end{aligned}
$$

Step 5 (Control of $\left\|h \nabla Z^{n}\right\|_{L^{2}(\Omega)}$ and $\left.\left\|Y^{n}\right\|_{L^{2}(\Omega)}\right)$. Combining (5.20) and (5.21), and setting $\epsilon=\frac{1}{8 c_{0}^{2}}$, we can finally rewrite (5.19) as follows:

$$
\begin{aligned}
\lambda^{2}\left\|h \nabla Z^{n}\right\|_{L^{2}}^{2} \leq c c_{M}^{2} g_{E} \lambda^{2(1+\alpha)} & \delta t_{n}^{2(1-\alpha)}\left\|U^{0}\right\|_{L^{2}(\Omega)}^{2} \\
& +c^{\prime}\left(c_{i}^{2} c_{M}+c_{M}^{-1}\right) \lambda^{3} \delta t_{n}\left\|\sqrt{\nu^{n}} \nabla W^{n}\right\|_{L^{2}(\Omega)}^{2} .
\end{aligned}
$$

We now combine the above estimate with (5.17) to arrive at

$$
\begin{aligned}
\left\|Y^{n}\right\|_{L^{2}}^{2} \leq c c_{M}^{2} g_{E} \lambda^{2(1+\alpha)} & \delta t_{n}^{2(1-\alpha)}\left\|U^{0}\right\|_{L^{2}(\Omega)}^{2} \\
& +c^{\prime} \lambda\left(1+\lambda^{2}\right)\left(c_{i}^{2} c_{M}+c_{M}^{-1}\right) \delta t_{n}\left\|\sqrt{\nu^{n}} \nabla W^{n}\right\|_{L^{2}(\Omega)}^{2} .
\end{aligned}
$$


Step 6. We now conclude. Upon observing that

$$
\begin{aligned}
\left|\left(Y^{n}, U^{n+1}-U^{n}\right)_{\Omega}\right| & =\left|\left\|Y^{n}\right\|_{L^{2}(\Omega)}^{2}+2\left(Y, W^{n}-U^{n}\right)_{\Omega}\right| \\
& \leq\left\|Y^{n}\right\|_{L^{2}(\Omega)}^{2}+\left\|Y^{n}\right\|_{L^{2}(\Omega)}\left\|Z^{n}\right\|_{L^{2}(\Omega)} .
\end{aligned}
$$

and by using the estimates (5.22) and (5.23) in (5.14), we infer that

$$
\begin{aligned}
\| U^{n+1} & \left\|_{L^{2}(\Omega)}^{2}-\right\| U^{n}\left\|_{L^{2}(\Omega)}^{2}+2 \delta t_{n}\right\| \sqrt{\nu^{n}} \nabla W^{n} \|_{L^{2}(\Omega)}^{2} \\
\leq & c c_{M}^{2} g_{E} \lambda^{2(1+\alpha)} \delta t_{n}^{2(1-\alpha)}\left\|U^{0}\right\|_{L^{2}(\Omega)}^{2} \\
& +c^{\prime} \lambda\left(1+\lambda^{2}\right)\left(c_{i}^{2} c_{M}+c_{M}^{-1}\right) \delta t_{n}\left\|\sqrt{\nu^{n}} \nabla W^{n}\right\|_{L^{2}(\Omega)}^{2} .
\end{aligned}
$$

Upon further restricting $\Lambda_{0}$ so that $c^{\prime} \Lambda_{0}\left(1+\Lambda_{0}^{2}\right)\left(c_{i}^{2} c_{M}+c_{M}^{-1}\right) \leq 1$, we conclude by using the usual telescoping argument.

Remark 5.3 (Restriction on $\alpha$ ). The stability restriction $\alpha<\frac{1}{2}$ in Theorem 5.1 seems to be technical. We conjecture again that Theorem 5.1 should hold in the range $\alpha \in[0,1]$.

\section{Discussion on Entropies}

The method discussed above bears some resemblance to the residual-based shock capturing techniques from [15]23 when $E(u)=u$. The present method is, however, significantly different from that in [15,23, in the sense that the viscosity is scaled differently, it is not allowed to exceed the first-order viscosity $c_{M}\|h \boldsymbol{\beta}\|_{L^{\infty}(K)}$, the time stepping is explicit, and our analysis does not require any sort of additional linear stabilization to work properly (Galerkin-Least-Squares, streamline diffusion [16], SUPG [3], Discontinuous Galerkin [17] or edge stabilization [6]). Our analysis is similar in spirit to that of [4], where convergence of a class of nonlinear viscosity methods for the one-dimensional Burgers equation is performed without using any type of linear stabilization. This idea was later applied to viscoelastic systems in [2]. However, our work differs from [4] in that the viscosity is built differently and the time is kept continuous in [4].

We illustrate the method on the inviscid Burgers equation in Figure 2 The domain is periodic, $\Omega=(0,1)$, the initial data is $u_{0}(x)=\sin (2 \pi x)$. The computation is done with continuous piecewise linear finite elements and RK2 time stepping (the Heun and the midpoint method give similar results). The solution is shown at $T=0.25$. The displayed results have been obtained with the residual viscosity, $E(u)=u$, and the square entropy $E(u)=\frac{1}{2} u^{2}$. We observe that the method performs very well in both cases and the viscosity focuses in the shock (note that the viscosity field in displayed in log scale).

In some cases it may be beneficial to use nonlinear entropies like $E(u)=|u-c|^{\gamma}$, $\gamma>1$. Although we have numerically observed that the method performs well with these entropies, we have not yet been able to prove stability. To motivate the use of higher-order entropies even for the linear transport equation, $\mathbf{f}(u)=\boldsymbol{\beta} u$, we show in Figure 3 numerical tests on the transport equation in the unit disk $\Omega=\left\{(x, y) \in \mathbb{R}^{2}, \sqrt{x^{2}+y^{2}}<1\right\}$ using the entropy viscosity method with three different entropies: $E(u)=u-\frac{1}{2}$ (Figure 3(a)), $E(u)=\left(u-\frac{1}{2}\right)^{2}$ (Figure 33(b)), and $E(u)=\left(u-\frac{1}{2}\right)^{30}$ (Figure 3 (c)). The velocity field is a solid rotation of angular velocity $2 \pi$, i.e., $\boldsymbol{\beta}=2 \pi(-y, x)$. The initial field is $u_{0}(\mathbf{x})=1$ if $\mathbf{x}$ is in the disk of radius 0.5 centered at $(0.6,0)$ and $u_{0}(\mathbf{x})=0$ otherwise. The space approximation 
is done on a mesh composed of $25901 \mathbb{P}_{2}$ nodes $(h \approx 0.025)$. The time stepping is done with the SSP RK3 method. The solution is computed at $T=10$, i.e., after 10 revolutions. This example shows that the higher the nonlinearity in the entropy the better the performance of the method when applied to the linear transport equation with piecewise constant data (at least in the eyeball-norm).

Finally, we would like to emphasis once again that the choice of entropy viscosity to be used is problem-dependent. It may happen that for problems with nonconvex fluxes more than one entropy may have to be used to construct the viscosity.

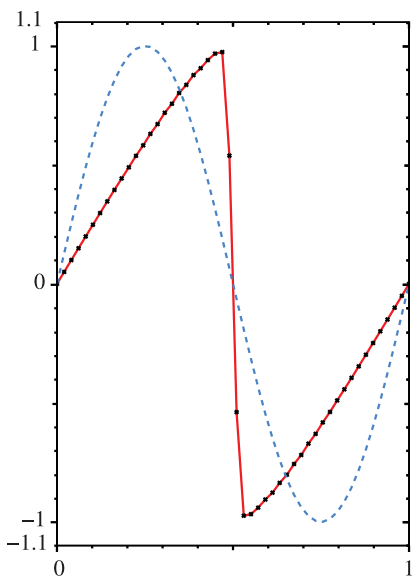

(a) $E(u)=u$

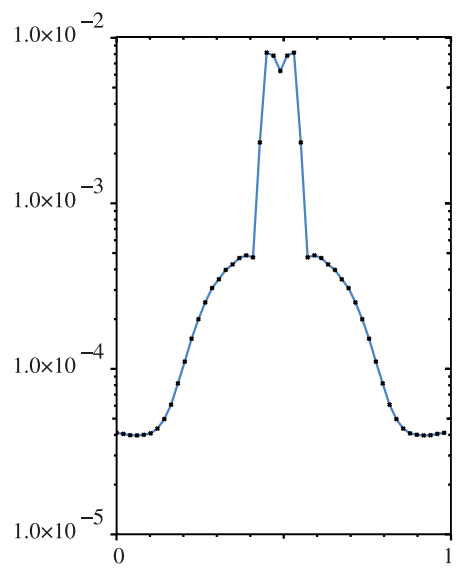

(c) $E(u)=u$

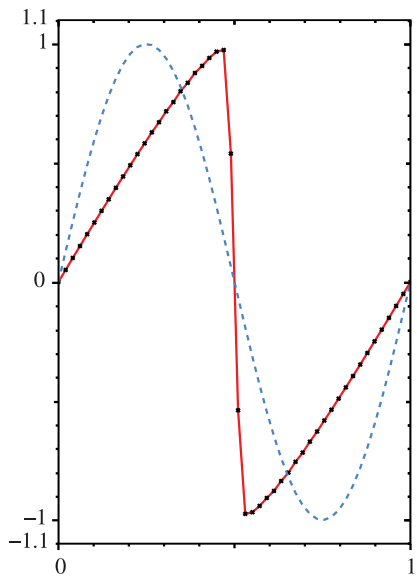

(b) $E(u)=\frac{1}{2} u^{2}$

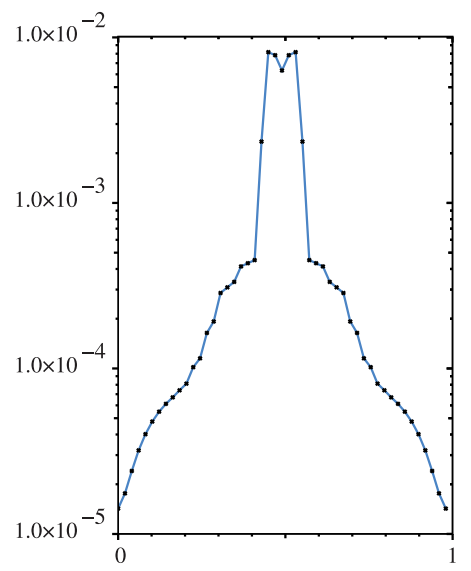

(d) $E(u)=\frac{1}{2} u^{2}$

Figure 2. Burgers equation, $\mathbb{P}_{1}$ continuous finite elements, RK3, 50 elements. 


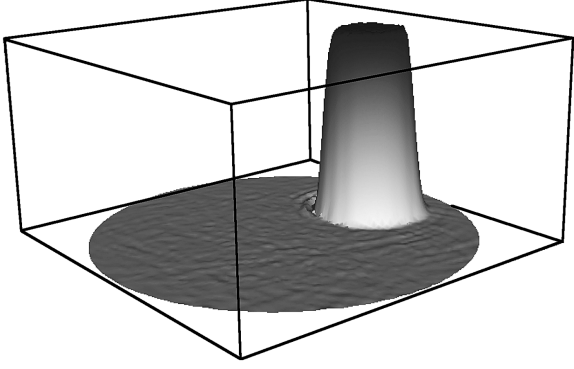

(a) $E(u)=2 u-1$

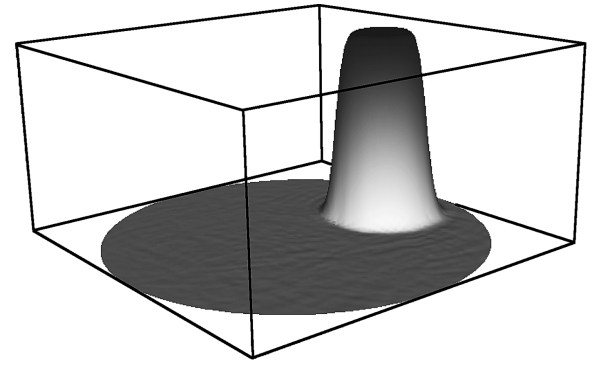

(b) $E(u)=(2 u-1)^{2}$

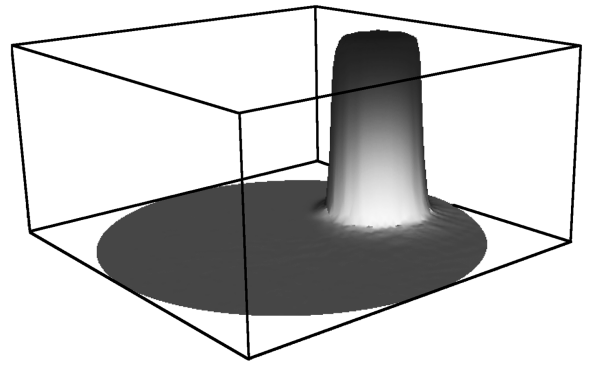

(c) $E(u)=(2 u-1)^{30}$

FiguRE 3. Tests on the linear transport equation with three different entropies.

\section{REFERENCES}

[1] C. Bardos, A. Y. le Roux, and J.-C. Nédélec, First order quasilinear equations with boundary conditions, Comm. Partial Differential Equations 4 (1979), no. 9, 1017-1034, DOI 10.1080/03605307908820117. MR542510 (81b:35052)

[2] Andrea Bonito and Erik Burman, A continuous interior penalty method for viscoelastic flows, SIAM J. Sci. Comput. 30 (2008), no. 3, 1156-1177, DOI 10.1137/060677033. MR2398860 (2009m:76010)

[3] Alexander N. Brooks and Thomas J. R. Hughes, Streamline upwind/Petrov-Galerkin formulations for convection dominated flows with particular emphasis on the incompressible Navier-Stokes equations, Comput. Methods Appl. Mech. Engrg. 32 (1982), no. 1-3, 199-259, DOI 10.1016/0045-7825(82)90071-8. FENOMECH '81, Part I (Stuttgart, 1981). MR679322 (83k:76005)

[4] Erik Burman, On nonlinear artificial viscosity, discrete maximum principle and hyperbolic conservation laws, BIT 47 (2007), no. 4, 715-733, DOI 10.1007/s10543-007-0147-7. MR2358367(2008m:76071)

[5] Erik Burman, Alexandre Ern, and Miguel A. Fernández, Explicit Runge-Kutta schemes and finite elements with symmetric stabilization for first-order linear PDE systems, SIAM J. Numer. Anal. 48 (2010), no. 6, 2019-2042, DOI 10.1137/090757940. MR2740540(2012a:65231)

[6] Erik Burman and Peter Hansbo, Edge stabilization for Galerkin approximations of convection-diffusion-reaction problems, Comput. Methods Appl. Mech. Engrg. 193 (2004), no. 15-16, 1437-1453, DOI 10.1016/j.cma.2003.12.032. MR2068903 (2005d:65186)

[7] Sigal Gottlieb, Chi-Wang Shu, and Eitan Tadmor, Strong stability-preserving high-order time discretization methods, SIAM Rev. 43 (2001), no. 1, 89-112 (electronic), DOI 10.1137/S003614450036757X. MR1854647 (2002f:65132) 
[8] Jean-Luc Guermond, On the use of the notion of suitable weak solutions in CFD, Internat. J. Numer. Methods Fluids 57 (2008), no. 9, 1153-1170, DOI 10.1002/fld.1853. MR2435087 (2009j:76064)

[9] Jean-Luc Guermond and Richard Pasquetti, Entropy-based nonlinear viscosity for Fourier approximations of conservation laws, C. R. Math. Acad. Sci. Paris 346 (2008), no. 13-14, 801-806, DOI 10.1016/j.crma.2008.05.013 (English, with English and French summaries). MR2427085(2009d:65130)

[10] Jean-Luc Guermond and Richard Pasquetti, Entropy viscosity method for high-order approximations of conservation laws, Spectral and High Order Methods for Partial Differential Equations, Selected papers from the ICOSAHOM '09 conference (Jan S. Hesthaven and Einar M. Ranquist, eds.), Lecture Notes in Computational Science and Engineering, vol. 76, Springer-Verlag, Heidelberg, 2011, pp. 411-418.

[11] Jean-Luc Guermond, Richard Pasquetti, and Bojan Popov, Entropy viscosity method for nonlinear conservation laws, J. Comput. Phys. 230 (2011), no. 11, 4248-4267, DOI 10.1016/j.jcp.2010.11.043. MR2787948 (2012h:65216)

[12] Ami Harten, Björn Engquist, Stanley Osher, and Sukumar R. Chakravarthy, Uniformly highorder accurate essentially nonoscillatory schemes. III, J. Comput. Phys. 71 (1987), no. 2, 231-303, DOI 10.1016/0021-9991(87)90031-3. MR897244(90a:65199)

[13] Ami Harten and Stanley Osher, Uniformly high-order accurate nonoscillatory schemes. I, SIAM J. Numer. Anal. 24 (1987), no. 2, 279-309, DOI 10.1137/0724022. MR881365 (90a:65198)

[14] Amiram Harten, Peter D. Lax, and Bram van Leer, On upstream differencing and Godunovtype schemes for hyperbolic conservation laws, SIAM Rev. 25 (1983), no. 1, 35-61, DOI 10.1137/1025002. MR693713 (85h:65188)

[15] Thomas J. R. Hughes and Michel Mallet, A new finite element formulation for computational fluid dynamics. IV. A discontinuity-capturing operator for multidimensional advectivediffusive systems, Comput. Methods Appl. Mech. Engrg. 58 (1986), no. 3, 329-336, DOI 10.1016/0045-7825(86)90153-2. MR865672(89j:76015c)

[16] Claes Johnson, Uno Nävert, and Juhani Pitkäranta, Finite element methods for linear hyperbolic problems, Comput. Methods Appl. Mech. Engrg. 45 (1984), no. 1-3, 285-312, DOI 10.1016/0045-7825(84)90158-0. MR759811 (86a:65103)

[17] C. Johnson and J. Pitkäranta, An analysis of the discontinuous Galerkin method for a scalar hyperbolic equation, Math. Comp. 46 (1986), no. 173, 1-26, DOI 10.2307/2008211. MR.815828 (88b:65109)

[18] Claes Johnson, Anders Szepessy, and Peter Hansbo, On the convergence of shock-capturing streamline diffusion finite element methods for hyperbolic conservation laws, Math. Comp. 54 (1990), no. 189, 107-129, DOI 10.2307/2008684. MR995210 (90j:65118)

[19] S. N. Kružkov, First order quasilinear equations with several independent variables., Mat. Sb. (N.S.) 81 (123) (1970), 228-255 (Russian). MR0267257 (42 \#2159)

[20] Haim Nessyahu and Eitan Tadmor, Nonoscillatory central differencing for hyperbolic conservation laws, J. Comput. Phys. 87 (1990), no. 2, 408-463, DOI 10.1016/0021-9991(90)90260-8. MR 1047564 (91i:65157)

[21] Bojan Popov and Ognian Trifonov, Order of convergence of second order schemes based on the minmod limiter, Math. Comp. 75 (2006), no. 256, 1735-1753, DOI 10.1090/S0025-571806-01875-8. MR2240633(2008a:65153)

[22] J. Smagorinsky, General circulation experiments with the primitive equations, part i: the basic experiment, Monthly Wea. Rev. 91 (1963), 99-152.

[23] Anders Szepessy, Convergence of a shock-capturing streamline diffusion finite element method for a scalar conservation law in two space dimensions, Math. Comp. 53 (1989), no. 188, 527-545, DOI 10.2307/2008718. MR979941(90h:65156)

[24] J. Von Neumann and R. D. Richtmyer, A method for the numerical calculation of hydrodynamic shocks, J. Appl. Phys. 21 (1950), 232-237. MR0037613 (12,289b)

[25] Qiang Zhang and Chi-Wang Shu, Stability analysis and a priori error estimates of the third order explicit Runge-Kutta discontinuous Galerkin method for scalar conservation laws, SIAM J. Numer. Anal. 48 (2010), no. 3, 1038-1063, DOI 10.1137/090771363. MR2669400 (2011g:65205) 
[26] Valentin Zingan, Jean-Luc Guermond, Jim Morel, and Bojan Popov, Implementation of the entropy viscosity method with the discontinuous galerkin method, Computer Methods in Applied Mechanics and Engineering 253 (2013), 479-490. MR3002806

Department of Mathematics, Texas A\&M University, College Station, Texas 77843 E-mail address: bonito@math.tamu.edu).

Department of Mathematics, Texas A\&M University, College Station, Texas 77843. On leave from LIMSI, UPRR 3251 CNRS, BP 133, 91403 Orsay Cedex, France

E-mail address: guermond@math.tamu.edu

Department of Mathematics, Texas A\&M University, College Station, Texas 77843

E-mail address: popov@tamu.edu 